\title{
Koszul duality theory for operads over Hopf algebras
}

\author{
Olivia Bellier
}

\begin{abstract}
The transfer of the generating operations of an algebra to a homotopy equivalent chain complex produces higher operations. The first goal of this paper is to describe precisely the higher structure obtained when the unary operations commute with the contracting homotopy. To solve this problem, we develop the Koszul duality theory of operads in the category of modules over a cocommutative Hopf algebra. This gives rise to a simpler category of homotopy algebras and infinity morphisms, which allows us to get a new description of the homotopy category of algebras over such operads. The main example of this theory is given by Batalin-Vilkovisky algebras.
\end{abstract}

18D50, 18G55; 16W30, 55P48

\section{Introduction}

Homotopy transfer In homotopical algebra, one problem is to know how algebraic structures behave under homotopy equivalences. For instance, the product of a differential graded associative algebra induces, on a homotopy equivalent chain complex, a product which is not associative in general. However, Kadeishvili [16] proved that, in this case, the homotopy equivalent chain complex carries higher operations, in addition to the transferred product. These operations endow it with a homotopy associative algebra structure, also known as $A_{\infty}$-algebra, defined by Stasheff in [25]. This is one of the first examples of a homotopy transfer theorem (HTT). An HTT was also proved for Lie algebras, for commutative algebras, and more generally, for other types of algebras using the theory of operads; see Loday and Vallette [21, Section 10.3].

Batalin-Vilkovisky algebras The theory we develop in this paper is motivated by the example of Batalin-Vilkovisky algebras. These algebras play an important role in geometry, topology and mathematical physics; see for instance Batalin and Vilkovisky [2], Barannikov and Kontsevich [1], Manin [22], Chas and Sullivan [6], Getzler [11] and Lian and Zuckerman [19]. In brief, a BV-algebra structure is given by a commutative product, a Lie bracket and a unary square-zero BV-operator, satisfying some compatibility relations. An HTT for BV-algebras was given by Galvez-Carillo, Tonks and Vallette [10] in terms of a new notion of homotopy BV-algebra. This structure is very rich, and at the same time very intricate. 
However, when the BV-operator commutes with the underlying contracting homotopy, it induces a unary operator which still squares to zero. In this case, we have that there are no higher operations arising from the BV-operator when we apply the HTT of [10]. It is natural to ask what is the precise structure to which it reduces. In this paper, we solve this question as follows: we insert the square-zero operator in the underlying category of chain complexes and, then, we work with operads in this category. This implies that we have to work with operads in the category of modules over a cocommutative Hopf algebra. For instance, to encode the category of BV-algebras, we use the operad of Gerstenhaber algebras, which is enriched with an action of the dual numbers Hopf algebra.

Relative Koszul duality Salvatore and Wahl [24] began the study of operads with an action of a Hopf algebra. Here, we go even further and we extend the classical Koszul duality theory to this framework in Section 3.3. At each step of the operadic theory, we show that all the objects can be enriched with a compatible action of a Hopf algebra and we prove that the results still hold in this context. In particular, we extend the bar-cobar constructions in Section 3.2. We define a new notion of homotopy algebras and their infinity morphisms in Section 4.1, in order to prove a new homotopy transfer theorem in Section 4.2. We also extend the results of the homotopy theory of algebras over an operad to the context of algebras over an $H$-operad in Section 4.3.

Theorem A Let $H$ be a cocommutative Hopf algebra and let $\mathcal{P}$ be a Koszul operad in the category of $\mathrm{H}$-modules. We consider two homotopy equivalent chain complexes in the category of $H$-modules, such that one of them is endowed with a compatible $\mathcal{P}$-algebra structure. Then the second chain complex is endowed with a $\mathcal{P}_{\infty}$-algebra structure compatible with the $H$-action.

The main example of two homotopy equivalent chain complexes is given by a differential graded algebra and its homology groups. In this case, being homotopy equivalent in the category of $H$-modules means just that the contracting homotopy and the $H$-action have to commute.

The homotopy category is obtained by localizing the initial category with respect to the quasi-isomorphisms. The $\infty$-morphisms provide a different description of the homotopy category of algebras over an operad. It is easier to deal with this description since the $\infty$-morphisms admit a homotopy inverse; see Vallette [27]. Encoding a category of algebras with an operad in $H$-modules, we get a new description of the associated homotopy category, using simpler $\infty$-morphisms, as follows. 
Theorem B Let $H$ be a cocommutative Hopf algebra and let $\mathcal{P}$ be a Koszul operad in the category of $H$-modules. The homotopy category of $\mathrm{dg} \mathcal{P}$-algebras in $\mathrm{H}$-modules is equivalent to the homotopy category of $\mathcal{P}$-algebras in $H$-modules, together with their $\infty-H$-morphisms.

In the case of BV-algebras, we define a simpler category of homotopy BV-algebras: a strict homotopy BV-algebra is a homotopy Gerstenhaber algebra together with a compatible square-zero unary operator action. Theorem A implies that, when the BVoperator and the contracting homotopy commute, the transferred homotopy BV-algebra structure reduces exactly to a strict homotopy $\mathrm{BV}$-algebra. Theorem $\mathrm{B}$ gives a new description of the homotopy category of $\mathrm{BV}$-algebras. We also get a new way to prove the existence of a zigzag of quasi-isomorphisms of BV-algebras, which could help to study the mirror symmetry conjecture by Kontsevich [17]; see Cao and Zhou [4; 5].

Related literature The idea to put unary operations in the underlying category is already used by Ginzburg and Kapranov [14], but not exactly in the same way as we do. We both put the algebra of all the arity one operations in the underlying category, while removing them from the operad. In [14], they keep track of their action using the tensor product over this algebra. Instead, we also remove from the operad all the operations made up of the unary ones, and we keep track of the action of these operations via the module structure over their algebra. Thus, we can still work with the tensor product over the ground field. So, homological results hold in our context, while, in [14], one needs the algebra of unary operations to be semisimple. Nevertheless, we need this algebra to form a Hopf algebra. Moreover, since Ginzburg and Kapranov remove only the unary operations from the operad, their homotopy algebra structure is much bigger than ours.

There are so far two ways of proving an HTT. On the one hand, one can prove it with model category arguments by Berger and Moerdijk [3]. This relies on a compatibility between the monoidal and the model structures of the underlying category. But, to the best of our knowledge, there is not yet a monoidal model category structure on the category of modules over a cocommutative Hopf algebra together with the tensor product over the ground field, such that the weak equivalences are the quasi-isomorphisms. On the other hand, one can prove an HTT with explicit formulae using the Koszul duality theory for operads, which enables one to prove formality results at the level of algebras for instance. So, we chose this latter method to prove an HTT for operads over Hopf algebras.

Layout In the second section, we describe the notion of operads in the category of modules over a Hopf algebra and the associated objects. We give some examples of algebras over such operads. In Section 3, we extend the classical Koszul duality to this 
framework. In Section 4, we prove an HTT for algebras over Koszul operads in this context, and we apply it to the example of Batalin-Vilkovisky algebras. Furthermore, we study the homotopy theory of such algebras.

Setting Throughout this paper, we work over a field $\mathbb{K}$ of characteristic zero and unadorned tensor products are over $\mathbb{K}$. All the $\mathbb{S}$-modules we consider are reduced, that is the arity zero space is reduced to zero.

\section{Operads over Hopf algebras}

\subsection{The monoidal category of symmetric modules over a cocommutative Hopf algebra}

We construct the monoidal category of (differential graded) $\mathbb{S}$-modules over a cocommutative Hopf algebra, following the example of $\mathbb{S}$-modules given by Loday and Vallette [21, Section 5.1]. We refer to the book of Sweedler [26] for more details on Hopf algebras.

\subsubsection{Recollection on the symmetric monoidal closed category of modules over a}

Hopf algebra Let $(H, \mu, \Delta, u, \varepsilon)$ be a cocommutative bialgebra, where $(\mu, u)$ and $(\Delta, \varepsilon)$ are respectively the unital associative algebra structure and the counital cocommutative coalgebra structure. We will denote $1_{H}:=u\left(1_{\mathbb{K}}\right)$. Then, we have $\varepsilon\left(1_{H}\right)=1_{\mathbb{K}}$ and $H \cong \operatorname{Ker}(\varepsilon: H \rightarrow \mathbb{K}) \oplus \mathbb{K} 1_{H}$.

We define the iterated comultiplication $\Delta^{n}: H \rightarrow H^{\otimes n+1}$ by $\Delta^{0}=\mathrm{Id}, \Delta^{1}=\Delta$ and

$$
\Delta^{n}:=(\Delta \otimes \operatorname{Id} \otimes \cdots \otimes \operatorname{Id}) \circ \Delta^{n-1} .
$$

The category $(H-\mathrm{Mod}, \otimes, \mathbb{K})$ of left- $H$-modules is a symmetric monoidal category where the structure of the $H$-module is given as follows:

- On $\mathbb{K}$, it is defined by the map $H \otimes \mathbb{K} \stackrel{\varepsilon \otimes \mathbb{K}}{\longrightarrow} \mathbb{K} \otimes \mathbb{K} \longrightarrow \mathbb{K}$ where the last map is the scalar multiplication.

- On the tensor product of two $H$-modules $M$ and $N$, it is defined by

$$
h .(m \otimes n)=h_{(0)} \cdot m \otimes h_{(1)} . n, \quad \forall h \in H, \forall m \in M, \forall n \in N,
$$

where $\Delta(h)=h_{(0)} \otimes h_{(1)}$, under the sumless Sweedler notation. The Hopf compatibility relation ensures that it is a left $H$-action. 
We assume moreover that $H$ is a Hopf algebra, that is $H$ is equipped with a linear map $S: H \rightarrow H$, called the antipode, which is an inverse of the identity for the convolution product $\star$ :

$$
S \star \operatorname{Id}=u \varepsilon=\operatorname{Id} \star S
$$

Then, for any $H$-modules $M$ and $N$, the vector space $\operatorname{Hom}_{\mathbb{K}}(M, N)$ carries an $H$-module structure, given by

$$
\begin{aligned}
H \otimes \operatorname{Hom}_{\mathbb{K}}(M, N) & \rightarrow \operatorname{Hom}_{\mathbb{K}}(M, N), \\
h \otimes f & \mapsto h . f: m \mapsto h_{(0)} \cdot f\left(S\left(h_{(1)}\right) . m\right) .
\end{aligned}
$$

Lemma 2.1 The category of $H$-modules is closed.

Proof For any triple $(A, B, C)$ of $H$-modules, the map

$$
\begin{aligned}
\operatorname{Hom}(A \otimes B, C) & \rightarrow \operatorname{Hom}(A, \operatorname{Hom}(B, C)), \\
f & \mapsto(a \mapsto f(a \otimes-)),
\end{aligned}
$$

is well defined. It is easy to check that it is a natural isomorphism of $H$-modules.

The previous results extend to the category of differential graded modules.

Proposition 2.2 We have that the category (dg $H$-Mod, $\otimes, \mathbb{K}$ ) of differential graded left-H-modules is a symmetric monoidal closed category, where $\mathbb{K}$ is concentrated in degree 0 .

Example Consider the graded algebra $D:=\mathbb{K}[\delta] /\left(\delta^{2}\right)$ of dual numbers, where $\delta$ is of degree +1 . It is a cocommutative Hopf algebra where the comultiplication $\Delta: D \rightarrow D \otimes D$ and the antipode $S: D \rightarrow D$ are respectively given by

$$
\begin{gathered}
\Delta(\delta):=1 \otimes \delta+\delta \otimes 1, \\
S(\delta):=-\delta .
\end{gathered}
$$

A $D$-module is simply a graded vector space endowed with a map of degree +1 that squares to zero, ie a cochain complex.

2.1.2 The monoidal category of $\mathbb{S}-\boldsymbol{H}$-modules The notion of $\mathbb{S}$-modules can be defined in any symmetric monoidal category. We make explicit these objects when the underlying category is equal to the category of $H$-modules. 
Definition 2.3 An $\mathbb{S}-H$-module $M$ is an $\mathbb{S}$-module $M=\{M(n)\}_{n \in \mathbb{N}}$, such that each $M(n)$ has a left- $H$-module structure which commutes with the $\mathbb{S}_{n}$-module structure. For $\mu \in M(n)$, the integer $n$ is called the arity of $\mu$ and $\mu$ is called an $n$-ary operation.

A morphism of $\mathbb{S}-H$-modules $f: M \rightarrow N$ is a morphism of $\mathbb{S}$-modules such that each $f_{n}: M(n) \rightarrow N(n)$ is $H$-equivariant.

We denote the associated category by $\mathbb{S}-H-$ Mod.

In particular, each $M(n)$ is a $\left(H, \mathbb{S}_{n}\right)$-bimodule and each $f_{n}: M(n) \rightarrow N(n)$ is a morphism of $\left(H, \mathbb{S}_{n}\right)$-bimodules.

Definition 2.4 For any $\mathbb{S}-H$-modules $M$ and $N$, their tensor product is the $\mathbb{S}-H-$ module $M \otimes N$ defined by

$$
(M \otimes N)(n):=\bigoplus_{i+j=n} \operatorname{Ind}_{\mathbb{S}_{i} \times \mathbb{S}_{j}}^{\mathbb{S}_{n}} M(i) \otimes N(j),
$$

where the action of $H$ is induced by the $H$-module structure on the tensor product of two $H$-modules. Since $H$ is cocommutative, the tensor product of $H$-modules is symmetric.

Proposition 2.5 The tensor product of $\mathbb{S}-H$-modules is associative with unit the $\mathbb{S}-H$-module $(\mathbb{K}, 0,0, \ldots)$.

Proof By [21, Proposition 5.1.5], we have an isomorphism of associativity of the underlying $\mathbb{S}$-modules. It is a morphism of $H$-modules by coassociativity of the comultiplication.

Definition 2.6 For any $\mathbb{S}-H$-modules $M$ and $N$, their composite product is the $\mathbb{S}-H$-module $M \circ N$ defined by

$$
M \circ N:=\bigoplus_{k \geq 0} M(k) \otimes_{\mathbb{S}_{k}} N^{\otimes k}
$$

Here $N^{\otimes k}$ stands for the tensor product of $k$ copies of the $\mathbb{S}-H$-module $N$.

For any pair of morphisms of $\mathbb{S}-H$-modules $f: M \rightarrow N$ and $g: M^{\prime} \rightarrow N^{\prime}$, their composite product is the morphism of $\mathbb{S}-H$-modules $f \circ g: M \circ N \rightarrow M^{\prime} \circ N^{\prime}$ given explicitly by the formula

$$
f \circ g\left(\mu ; v_{1}, \ldots, v_{k}\right):=\left(f(\mu) ; g\left(v_{1}\right), \ldots, g\left(v_{k}\right)\right) .
$$


In arity $n$, we have

$$
(M \circ N)(n):=\bigoplus_{k \geq 0} M(k) \otimes \mathbb{S}_{k}\left(\bigoplus_{i_{1}+\cdots+i_{k}=n} \operatorname{Ind}_{\mathbb{S}_{i_{1}} \times \cdots \times \mathbb{S}_{i_{k}}}^{\mathbb{S}_{n}} N\left(i_{1}\right) \otimes \cdots \otimes N\left(i_{k}\right)\right)
$$

where the action of $H$ is induced by the $H$-module structure on tensor products of $H$-modules.

Proposition 2.7 The category of $\mathbb{S}-H$-modules ( $\mathbb{S}-H$-Mod, $\circ, I$ ) is a monoidal category, where the $\mathbb{S}-H$-module $I=(0, \mathbb{K}, 0, \ldots)$ concentrated in arity 1 is called identity $\mathbb{S}-H-$ module.

Proof By [21, Proposition 5.1.14], the category of $\mathbb{S}$-modules $(\mathbb{S}-$ Mod,,$I)$ is monoidal. Since the composite product of $\mathbb{S}-H$-modules is defined as the composite product of the underlying $\mathbb{S}$-modules endowed with the $H$-action induced by the comultiplication, the coassociativity and the counitarity of the comultiplication of $H$ imply that the isomorphisms of associativity and unit of the underlying $\mathbb{S}$-modules are compatible with the $H$-module structure.

As for $\mathbb{S}$-modules, the composite product of two $\mathbb{S}-H$-modules is not linear on the right-hand side. However, the infinitesimal composite product, defined in [21, Section 6.1.1], extends to the category $\mathbb{S}-H$-Mod to produce a product ${ }^{\circ}(1)$ which is linear on the right-hand side. Recall that the infinitesimal product is defined by

$$
M \circ_{(1)} N:=M \circ(I ; N),
$$

where, for any $\mathbb{S}$-modules $P, P_{1}$ and $P_{2}, P \circ\left(P_{1} ; P_{2}\right)$, is the $\mathbb{S}$-module

$$
\left(P \circ\left(P_{1} ; P_{2}\right)\right)(n)
$$

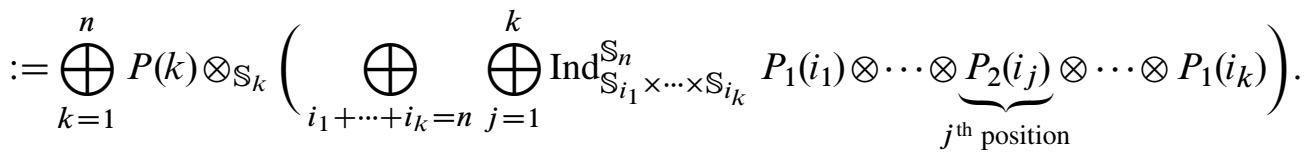

The infinitesimal objects $f \circ_{(1)} g, f \circ^{\prime} g, \gamma_{(1)}$ and $\Delta_{(1)}$, defined in [21, Section 6.1], also extend to the category $\mathbb{S}-H$-Mod.

\subsubsection{Differential graded framework}

Definition 2.8 A differential graded $\mathbb{S}-H$-module, or $\mathrm{dg} \mathbb{S}-H$-module for short, is a dg $\mathbb{S}$-module $(M, d)$ such that each $M(n)$ is an $H$ - $\mathbb{S}_{n}$-bimodule and the differential $d$ is compatible with the $H$-action. 
A morphism of differential graded $\mathbb{S}-H$-modules $f:\left(M, d_{M}\right) \rightarrow\left(N, d_{N}\right)$ is a morphism of the underlying dg $\mathbb{S}$-modules which is $H$-equivariant. We denote by $\operatorname{dg} \mathbb{S}-H$-Mod the category of dg $\mathbb{S}-H$-modules with their morphisms.

The objects described in the previous section extend to the differential graded framework. However, they now involve signs in their definition. For more details, we refer the reader to [21, Section 6.2].

We define the suspension and the desuspension of a graded $\mathbb{S}-H$-module as the suspension and the desuspension of its underlying graded $\mathbb{S}$-module respectively, where the action of $H$ is given by the counit of $H$.

Proposition 2.9 The category of ( $\mathrm{dg} \mathbb{S}-H-\mathrm{Mod}, \circ, I)$ is a monoidal category.

Proof This follows by combining Proposition 2.7 and [21, Proposition 6.2.4].

\subsection{Operad over a cocommutative Hopf algebra}

In [21, Section 5.2], the authors give a monoidal definition of an algebraic operad, that is an operad in the category of vector spaces. More generally, one can define the notion of operad in any monoidal category. Here, we point out the extra structure one gets when one considers operads in the category of $\mathbb{S}-H$-modules instead of just $\mathbb{S}$-modules.

\subsubsection{Monoidal definition}

Definition 2.10 An $H$-operad is a monoid $(\mathcal{P}, \gamma, \eta)$ in the monoidal category of $\mathbb{S}-$ $H$-modules. A morphism $\alpha: \mathcal{P} \rightarrow \mathcal{Q}$ of $H$-operads is a morphism of monoids in the category of $\mathbb{S}-H$-modules, that is a morphism of $\mathbb{S}-H$-modules which is compatible with the monoidal structures. We denote the category of $H$-operads by $\mathrm{Op}_{H}$.

Remark An $H$-operad is just an operad $(\mathcal{P}, \gamma, \eta)$, as defined in [21, Section 5.2.1], such that each $\mathcal{P}(n)$ has an $H$-action which makes $\mathcal{P}$ into an $\mathbb{S}-H$-module and such that the maps $\gamma$ and $\eta$ commute with the $H$-action. Furthermore, a morphism of $H$-operads is a morphism of the underlying operads which commutes with the action of $H$.

When $A$ is an $H$-module, the $\mathbb{S}$-module

$$
\operatorname{End}_{A}(n):=\operatorname{Hom}_{\mathbb{K}}\left(A^{\otimes n}, A\right)
$$


is endowed with an $H$-module structure, according to the previous section, which makes it into an $\mathbb{S}-H$-module. This structure is explicitly given by

$$
\begin{aligned}
H \otimes \operatorname{End}_{A}(n) & \rightarrow \operatorname{End}_{A}(n) \\
h \otimes f & \mapsto h . f:=a_{1} \otimes \cdots \otimes a_{n} \mapsto h_{(0)} \cdot f\left(S\left(h_{(1)}\right) \cdot a_{1}, \ldots, S\left(h_{(n)}\right) \cdot a_{n}\right) .
\end{aligned}
$$

Lemma 2.11 The triple $\left(\operatorname{End}_{A}, \gamma_{\text {End }_{A}}, \eta_{\operatorname{End}_{A}}: 1_{\mathbb{K}} \mapsto \operatorname{Id}_{A}\right)$, where $\gamma_{\text {End }_{A}}$ is the usual composition map given by $\gamma_{\operatorname{End}_{A}}\left(f ; g_{1}, \ldots, g_{k}\right):=f \circ\left(g_{1} \otimes \cdots \otimes g_{k}\right)$, is an $H$-operad.

Proof It is immediate to check End $A$ is an operad. So, we have to prove $\gamma_{\text {End }}$ and $\eta_{\text {End }_{A}}$ are $H$-equivariant maps. For $\left(f ; g_{1}, \ldots, g_{k}\right) \in \operatorname{End}_{A}(n), a_{1} \otimes \cdots \otimes a_{n} \in A^{\otimes n}$ and $h \in H$, we have

$$
\begin{aligned}
& \gamma_{\operatorname{End}_{A}}\left(h \cdot\left(f ; g_{1}, \ldots, g_{k}\right)\right)\left(a_{1} \otimes \cdots \otimes a_{n}\right) \\
& =h_{(0)} \cdot f\left(\bigotimes_{j=1}^{k}\left[S\left(h_{(n+j)(0)}\right) h_{(n+j)(1)}\right] \cdot g_{j}\left(\bigotimes_{l=1}^{i_{j}} S\left(h_{\left(i_{1}+\cdots+i_{j-1}+l\right)}\right) \cdot a_{i_{1}+\cdots+i_{j-1}+l}\right)\right) \\
& =h_{(0)} \cdot f\left(\bigotimes_{j=1}^{k} u \varepsilon\left(h_{(n+j)}\right) \cdot g_{j}\left(\bigotimes_{l=1}^{i_{j}} S\left(h_{\left(i_{1}+\cdots+i_{j-1}+l\right)}\right) \cdot a_{i_{1}+\cdots+i_{j-1}+l}\right)\right) \\
& =h_{(0)} \cdot\left(f \circ\left(g_{1} \otimes \cdots \otimes g_{k}\right)\left(S\left(h_{(1)}\right) \cdot a_{1} \otimes \cdots \otimes S\left(h_{(n)}\right) \cdot a_{n}\right)\right. \\
& =\left(h \cdot \gamma_{\operatorname{End}_{A}}\left(f ; g_{1}, \ldots, g_{k}\right)\right)\left(a_{1} \otimes \cdots \otimes a_{n}\right) .
\end{aligned}
$$

The $H$-equivariance of $\eta_{\operatorname{End}_{A}}$ follows from the $H$-module structure of $\mathbb{K}$ and from the fact that the antipode is the inverse of the identity for the convolution product.

Recall that the Hadamard product $M \otimes N$ of two $\mathbb{S}$-modules $M$ and $N$ is defined to be the following $\mathbb{S}$-module

$$
M \underset{\mathcal{H}}{\otimes} N(n):=M(n) \otimes N(n)
$$

Proposition 2.12 The Hadamard product of the underlying $\mathbb{S}$-modules of two $H$ operads, endowed with the $H$-module structure on the tensor product of two $\mathbb{S}-H$ modules, is an $H$-operad.

Proof Let $\mathcal{P}$ and $\mathcal{Q}$ be two $H$-operads. By [21, Section 5.3.3], we have to prove that the composition map given there is $H$-equivariant. This is the case since it is the composite of $H$-equivariant maps. 
Definition 2.13 An ideal $\mathcal{I}$ of an $H$-operad $\mathcal{P}$ is a sub- $\mathbb{S}-H$-module of $\mathcal{P}$ such that the composition $\gamma_{\mathcal{P}}\left(\mu ; v_{1}, \ldots, v_{k}\right)$ is in $\mathcal{I}$ as soon as one of the $\mu, v_{1}, \ldots, v_{k}$ is in $\mathcal{I}$. The quotient of an $H$-operad $\mathcal{P}$ by the ideal $\mathcal{I}$ is the $H$-operad $\mathcal{P} / \mathcal{I}$ given by

$$
(\mathcal{P} / \mathcal{I})(n):=\mathcal{P}(n) / \mathcal{I}(n), \quad \forall n \in \mathbb{N}
$$

where the composition map $\gamma_{\mathcal{P} / \mathcal{I}}$ is induced by $\gamma_{\mathcal{P}}$.

\subsubsection{The free $H$-operad}

Definition 2.14 A free $H$-operad over an $\mathbb{S}-H$-module $M$ is an $H$-operad $\mathcal{F} M$ equipped with a morphism $\eta_{M}: M \rightarrow \mathcal{F} M$ of $\mathbb{S}-H$-modules, which satisfies the following universal property.

Any morphism $f: M \rightarrow \mathcal{P}$ of $\mathbb{S}-H$-modules, where $\mathcal{P}$ is an $H$-operad, extends uniquely into a morphism $\tilde{f}: \mathcal{F} M \rightarrow \mathcal{P}$ of $H$-operads:

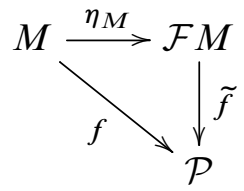

In other words, the functor $\mathcal{F}: \mathbb{S}-H$-Mod $\rightarrow \mathrm{Op}_{H}$ is left adjoint to the forgetful functor $\sqcup$ from $\mathrm{Op}_{H}$ to $\mathrm{S}-H-\mathrm{Mod}$.

Recall that the free operad $\mathcal{T} M$ over an $\mathbb{S}$-module $M$ admits the realization

$$
(\mathcal{T} M)(n) \cong \bigoplus_{\tau \in T^{\prime}(n)} \tau(M)
$$

where $T^{\prime}(n)$ denotes a set of representatives of isomorphisms classes of $n$-trees. For any tree $\tau$, the treewise tensor module $\tau(M)$, defined by Hoffbeck [15, Section 2.6], is given by

$$
\tau(M) \cong \bigotimes_{v \in V(\tau)} M\left(\left|I_{v}\right|\right),
$$

where $V(\tau)$ denotes the set of vertices of $\tau$ and where $I_{v}$ denotes the set of entries of the vertex $v$. Then, if $M$ is an $\mathbb{S}-H$-module, we have an $H$-module structure on the treewise tensor module $\tau(M)$, for any tree $\tau$ in $T^{\prime}(n)$. This action is given by the map

$$
\begin{aligned}
& H \otimes \tau(M) \rightarrow \tau(M), \\
& h \otimes m_{i_{1}} \cdots m_{i_{|V(\tau)|}} \mapsto h_{(0)} \cdot m_{i_{1}} \cdots h_{(|V(\tau)|-1)} \cdot m_{i_{|V(\tau)|}},
\end{aligned}
$$


where $\Delta^{|V(\tau)|-1}(h):=h_{(0)} \otimes \cdots \otimes h_{(|V(\tau)|-1)}$. It amounts to act on the labeling of each vertex of $\tau$ using the comultiplication of $H$.

Proposition 2.15 Let $M$ be an $\mathbb{S}-H$-module and $\underline{M}$ be its underlying $\mathbb{S}$-module. The free operad $\mathcal{T} \underline{M}$ endowed with the $H$-action described above is the free $H$-operad over $M$.

Proof The composition map $\gamma_{\mathcal{T} \underline{M}}$, which corresponds to the grafting of trees, is an $H$-equivariant map. Moreover, if a morphism $f: M \rightarrow \mathcal{P}$ of $\mathbb{S}$-modules is $H$ equivariant then so is $\tilde{f}: \mathcal{T} \underline{M} \rightarrow \mathcal{P}$.

As a consequence, the free $H$-operad inherits the weight of its underlying free operad. The weight of an operation $\mu$ in the free operad $\mathcal{T} M$ is defined as follows:

$$
\begin{aligned}
w(\mathrm{id}) & =0, \\
w(\mu) & =1, \quad \forall \mu \in M, \\
w\left(v ; v_{1}, \ldots, v_{k}\right) & =w(v)+w\left(v_{1}\right)+\cdots+w\left(v_{k}\right) .
\end{aligned}
$$

We denote by $\mathcal{T} M^{(r)}$ the $\mathbb{S}-H$-module of operations in $\mathcal{T}(M)$ of weight $r$.

Proposition 2.16 Let $M$ be an $\mathbb{S}$-module and $R$ be a sub-S-module of $\mathcal{T} M$. Then, the operad $\mathcal{T} M /(R)$, where $(R)$ is the ideal of $\mathcal{T} M$ generated by $R$, is an $H$-operad if and only if

- $M$ is an $\mathbb{S}-H$-module,

- $R$ is a sub-S-H-module of $\mathcal{T} M$.

In this case, the $H$-module structure is induced by the action on each vertex using the comultiplication of $H$.

Proof The proof by Salvatore and Wahl [24, Proposition 4.2] extends to our linear context.

\subsubsection{Algebra over an $\boldsymbol{H}$-operad}

Definition 2.17 Let $\mathcal{P}$ be an $H$-operad. An algebra over $\mathcal{P}$, or for short an $H-\mathcal{P}$ algebra, is an $H$-module $A$ equipped with an $H$-equivariant map $\gamma_{A}: \mathcal{P}(A) \rightarrow A$, which is compatible with the monoidal structure of $\mathcal{P}$. A morphism $f: A \rightarrow A^{\prime}$ of $H$-P-algebras is a morphism of $H$-modules which is compatible with $\gamma_{A}$ and $\gamma_{A^{\prime}}$. We denote by $H-\mathcal{P}-$ Alg the category of $H-\mathcal{P}$-algebras. 
If $A$ is an $H-\mathcal{P}$-algebra, then the map $\gamma_{A}$ is

$$
\gamma_{A}: \mathcal{P}(A)=\bigoplus_{n \geq 0} \mathcal{P}(n) \otimes_{\mathbb{S}_{n}} A^{\otimes n} \rightarrow A .
$$

For $\mu \in \mathcal{P}(n)$ and $a_{1} \otimes \cdots \otimes a_{n} \in A^{\otimes n}$, we denote $\gamma_{A}\left(\mu ; a_{1}, \ldots, a_{n}\right)$ simply by $\mu\left(a_{1}, \ldots, a_{n}\right)$.

Proposition 2.18 Let $\mathcal{P}$ be an $H$-operad. An $H$-P-algebra structure on an $H-$ module $A$ is equivalent to a morphism of $H$-operads $\mathcal{P} \rightarrow \operatorname{End}_{A}$.

Proof By [21, Proposition 5.2.13], it remains to prove that $\gamma_{A}: \mathcal{P}(n) \otimes A^{\otimes n} \rightarrow A$ is $H$-equivariant if and only if $\alpha: \mathcal{P}(n) \rightarrow \operatorname{End}_{A}(n)$ is $H$-equivariant, since the symmetric and the $H$ actions are compatible. This follows from the $H$-action on the space of morphisms of $H$-modules.

Proposition 2.19 [24] Let $\mathcal{P}$ be a graded $H$-operad. There exists a graded operad, the semidirect product $\mathcal{P} \rtimes H$, such that the category of $H$-P-algebras, that is $H$ modules with an action of the $H$-operad $\mathcal{P}$, is isomorphic to the category of $\mathcal{P} \rtimes H$ algebras, that is modules with an action of the operad $\mathcal{P} \rtimes H$.

The operad $\mathcal{P} \rtimes H$ is given by the $\mathbb{S}$-module defined by

$$
\mathcal{P} \rtimes H(n):=\mathcal{P}(n) \otimes H^{\otimes n},
$$

where $\mathbb{S}_{n}$ acts diagonally acting on $\mathcal{P}(n)$ and permuting the elements of $H^{\otimes n}$, together with the composition map defined by

$\gamma_{\mathcal{P} \rtimes H}\left((\mu \otimes h) ;\left(v_{1} \otimes g_{1}\right), \ldots,\left(v_{k} \otimes g_{k}\right)\right)$

$$
:=\gamma_{\mathcal{P}}\left(\mu ; h_{(0)}^{1} \cdot v_{1}, \ldots, h_{(0)}^{k} \cdot v_{k}\right) \otimes h_{(1)}^{1} \cdot g_{1} \otimes \cdots \otimes h_{(1)}^{k} \cdot g_{k},
$$

where $h=h^{1} \otimes \cdots \otimes h^{k}, g_{i}=g_{i}^{1} \otimes \cdots \otimes g_{i}^{n_{i}}$ and where $h^{i}$ acts on $g_{i}$ via the comultiplication of $H$. The unit of $\mathcal{P} \rtimes H$ is given by id $\otimes 1_{H}$.

\subsubsection{Cooperads over a cocommutative Hopf algebra}

Definition 2.20 An $H$-cooperad is a comonoid $(\mathcal{C}, \Delta, \eta)$ in the monoidal category of $\mathbb{S}-H$-modules. A morphism of $H$-cooperads $f: \mathcal{C} \rightarrow \mathcal{D}$ is a morphism of the underlying $\mathbb{S}-H$-modules compatible with the comonoidal structure of $\mathcal{C}$ and $\mathcal{D}$. We denote by Coop $_{H}$ the category of $H$-cooperads. There is an element id $\in \mathcal{C}(1)$ such that $\eta(\mathrm{id})=1_{\mathbb{K}}$ and which is called the identity cooperation.

An $H$-cooperad $\mathcal{C}$ is said to be coaugmented if there is a morphism $v: I \rightarrow \mathcal{C}$ of $H$-cooperads such that $\eta v=\operatorname{Id}_{I}$. 
The notion of $H$-cooperad is dual to that of $H$-operad. From the explicit description of the composite product of two $\mathbb{S}-H$-modules and the isomorphism between invariants and coinvariants, it follows that $\Delta$ is made up of $H$ - $\mathbb{S}_{n}$-bimodule morphisms:

$\Delta(n): \mathcal{C}(n) \rightarrow(\mathcal{C} \circ \mathcal{C})(n)=\bigoplus_{k=1}^{n} \mathcal{C}(k) \otimes_{\mathbb{S}_{k}}\left(\bigoplus_{i_{1}+\cdots+i_{k}=n} \operatorname{Ind}_{\mathbb{S}_{i_{1}} \times \cdots \times \mathbb{S}_{i_{k}}}^{\mathbb{S}_{n}} \mathcal{C}\left(i_{1}\right) \otimes \cdots \otimes \mathcal{C}\left(i_{k}\right)\right)$

Proposition 2.21 The Hadamard product of two $H$-cooperads carries an $H$-cooperad structure.

Proof Let $\mathcal{C}$ and $\mathcal{D}$ be two $H$-cooperads. By [21, Section 8.3.3], it remains to prove that the decomposition map given there is $H$-equivariant. This is the case since this decomposition map is the composite of $\Delta_{\mathcal{C}} \otimes \Delta_{\mathcal{D}}$ with maps of the type $\mathrm{Id} \otimes \cdots \otimes \tau \otimes \cdots \otimes \mathrm{Id}$, which are both $H$-equivariant.

Definition 2.22 Let $M$ be an $\mathbb{S}-H$-module such that $M(0)=0$. The cofree $H$ cooperad on $M$ is the $H$-cooperad, denoted by $\mathcal{T}^{c}(M)$, which is cofree in the category of conilpotent $H$-cooperads. It means that $\mathcal{T}^{c}(M)$ satisfies the following universal property.

For any morphism of $\mathbb{S}-H$-modules $\Phi: \mathcal{C} \rightarrow M$, from a conilpotent $H$-cooperad $\mathcal{C}$, such that $\Phi(\mathrm{id})=0$, there exists a unique morphism $\widetilde{\Phi}: \mathcal{C} \rightarrow \mathcal{T}^{c}(M)$ of $H$-cooperads whose corestriction to $M$ is equal to $\Phi$ :

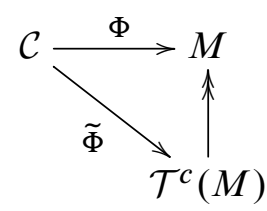

2.2.5 From operads to cooperads and vice versa In the classical case, the aritywise linear dual $\mathbb{S}$-module $\mathcal{C}^{*}=\left\{\operatorname{Hom}_{\mathbb{K}}(\mathcal{C}(n), \mathbb{K})\right\}_{n \in \mathbb{N}}$ associated to a cooperad $\mathcal{C}$ carries an operad structure. The unit is obtained by dualization of the counit $\eta_{\mathcal{C}}$ and the composition map is obtained by dualization of $\Delta_{\mathcal{C}}$ composed with the natural map from invariants to coinvariants given in [21, Section 5.1.21]. Since $H$ is a Hopf algebra, if $\mathcal{C}$ is moreover an $H$-cooperad then its aritywise dual has an $H$-operad structure. Equally, if $\mathcal{P}$ is an $H$-operad, such that each $\mathcal{P}(n)$ is finite dimensional, then its linear dual has an $H$-cooperad structure. We denote this cooperad by $\mathcal{P}^{c}$. 
2.2.6 Differential graded framework As for operads, the notion of $H$-operad extends to the differential graded framework. In particular, a differential graded $\mathrm{H}-$ operad is a monoid $\left(\mathcal{P}, d_{\mathcal{P}}, \gamma, \eta\right)$ in the monoidal category $(\mathrm{dg} \mathbb{S}-H$-Mod, $\circ, I)$, that is $\left(\mathcal{P}, d_{\mathcal{P}}\right)$ is a $\mathrm{dg} \mathbb{S}-H$-module and $(\mathcal{P}, \gamma, \eta)$ is an $H$-operad structure on $\mathcal{P}$, such that $\gamma$ and $\eta$ are morphisms of dg $\mathbb{S}-H$-modules. Moreover, the results on dg operads of [21, Section 6.3] can be extended to dg $H$-operads.

We denote by $\mathcal{S}$ (resp. $\mathcal{S}^{-1}$ ) the endomorphism $H$-operad associated to the graded $\mathbb{S}-H$-module $\mathbb{K} s$ (resp. $\mathbb{K} s^{-1}$ ), which is nothing but the $H$-module $\mathbb{K}$ concentrated in degree +1 (resp. in degree -1$)$. Note that we will denote by $\mathcal{S} M$ (resp. $\mathcal{S}^{-1} M$ ) the Hadamard product of $\mathcal{S}$ (resp. $\mathcal{S}^{-1}$ ) with any $\mathbb{S}-H$-module $M$.

\subsection{Examples}

2.3.1 Mixed chain complexes A mixed chain complex is a graded vector space $V=V \bullet$ endowed with two maps: a degree -1 map $d$ and a degree +1 map $\delta$, which both square to zero and anti-commute. One can see any mixed chain complex either as a dg-D-module, or as a dg $D-I$-algebra, where $I$ is the identity $D$-operad.

\subsubsection{Batalin-Vilkovisky algebras}

Definition A Gerstenhaber algebra is a differential graded vector space $\left(A, d_{A}\right)$ endowed with
$\diamond$ a symmetric binary product $\bullet$ of degree 0 ,
$\diamond$ a symmetric bracket $\langle-;-\rangle$ of degree +1 ,

such that $d_{A}$ is a derivation with respect to each of them and such that:

$\triangleright$ The product $\bullet$ is associative:

$$
((-\bullet-) \bullet-)=(-\bullet(-\bullet-)) \text {. }
$$

$\triangleright$ The bracket $\langle-;-\rangle$ satisfies the Jacobi identity

$$
\langle\langle-;-\rangle ;-\rangle+\langle\langle-;-\rangle ;-\rangle \cdot(123)+\langle\langle-;-\rangle ;-\rangle \cdot(321)=0 .
$$

$\triangleright$ The product $\bullet$ and the bracket $\langle-;-\rangle$ satisfy the Leibniz relation

$$
\langle-;-\bullet-\rangle=(\langle-;-\rangle \bullet-)+(-\bullet\langle-;-\rangle) \cdot(12) .
$$


A Batalin-Vilkovisky algebra, or BV-algebra for short, is a Gerstenhaber algebra $A$ endowed, in addition, with

$\diamond$ a unary operator $\Delta$ of degree +1 ,

such that $d_{A}$ anticommutes with $\Delta$ and such that:

- The operator satisfies $\Delta^{2}=0$.

- The bracket is the obstruction to $\Delta$ being a derivation with respect to the product $\bullet$ :

$$
\langle-;-\rangle=\Delta \circ(-\bullet-)-(\Delta(-) \bullet-)-(-\bullet \Delta(-))
$$

- The operator $\Delta$ is a graded derivation with respect to the bracket $\langle-;-\rangle$ :

$$
\Delta \circ(\langle-;-\rangle)+\langle\Delta(-) ;-\rangle+\langle-; \Delta(-)\rangle=0 \text {. }
$$

Let $\mathcal{G}$ be the operad encoding Gerstenhaber algebras. It is defined by generators and relations as

$$
\mathcal{G}:=\mathcal{T}(E) /(R)
$$

where $E=E(2):=\bullet \mathbb{K}_{2} \oplus\langle-;-\rangle \mathbb{K}_{2}$, with $\mathbb{K}_{2}$ the trivial representation of the symmetric group $\mathbb{S}_{2}$. The space of relations $R$ is the sub-S-module of $\mathcal{T}(E)$ generated by the relations $\triangleright$. The operad $\mathcal{B} \mathcal{V}$, encoding $\mathrm{BV}$-algebras, is then given by

$$
\mathcal{T}\left(E^{\prime}\right) /\left(R^{\prime}\right)
$$

where $E^{\prime}:=E \oplus \Delta \mathbb{K}$ and where $R^{\prime}$ is the sub-S-module of $\mathcal{T}\left(E^{\prime}\right)$ generated by the relations $\triangleright$ and

Lemma 2.23 The action of $D$ on the generators $\bullet$ and $\langle-;-\rangle$ of $\mathcal{G}$ is given by

$$
\begin{aligned}
\delta: \bullet \mapsto\langle-;-\rangle, & \mapsto\langle- \\
\langle-;-\rangle & \mapsto 0,
\end{aligned}
$$

and induces a $D$-operad structure on $\mathcal{G}$.

Proof It is clear that $E$ is an $\mathbb{S}-D$-module. Under the action of $\delta$, the associativity relation is sent to a linear combination of Leibniz relations, the Jacobi relation is sent to 0 and the Leibniz relation is sent to the Jacobi relation. Thus, $R$ is a sub-S $-D-$ module of $\mathcal{T}(E)$. We conclude with Proposition 2.16. 
Remark The $\delta$-action on the generators of $\mathcal{G}$ corresponds to the effect in homology of the action of the circle group $S^{1}$ on the little discs operad which is given by Getzler in [12, Section 4]. This structure induced in homology is made explicit by Salvatore and Wahl [24, Section 5].

Let $\mathcal{C}$ om denote the operad encoding commutative algebras and $\mathcal{L i e}_{1}$ denote the operad encoding Lie algebras with a bracket of degree +1 . As a consequence of a result by Markl [23, Theorem 2.7, Example 4.4], we have $\mathcal{G} \cong \mathcal{C}$ om $\circ \mathcal{L} i e_{1}$ as operads, under the distributive law

$$
\Lambda:\langle-;-\bullet-\rangle \mapsto(\langle-;-\rangle \bullet-)+(-\bullet\langle-;-\rangle) \cdot(12) .
$$

This isomorphism gives us a way to make explicit the $D$-operad structure on $\mathcal{G}$. As $\mathbb{S}$-modules, we have

$$
\mathcal{C} \text { om } \circ \mathcal{L} i e_{1}=\bigoplus_{k=1}^{n} \mathcal{C} \operatorname{Com}(t) \otimes \mathbb{S}_{t}\left(\bigoplus_{i_{1}+\cdots+i_{k}=n} \operatorname{Ind}_{\mathbb{S}_{i_{1}} \times \cdots \times \mathbb{S}_{i_{k}}}^{\mathbb{S}_{n}} \mathcal{L} i e_{1}\left(i_{1}\right) \otimes \cdots \otimes \mathcal{L i e}_{1}\left(i_{t}\right)\right) .
$$

The action of $\delta$ is compatible with the symmetric action, thus it is sufficient to give the

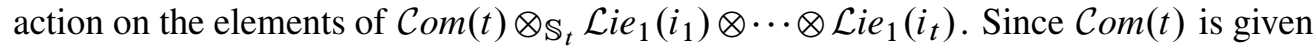
by the trivial representation on $\mathbb{K}$, an element in $\operatorname{Com}(t) \otimes_{\mathbb{S}_{t}} \mathcal{L i e}_{1}\left(i_{1}\right) \otimes \cdots \otimes \mathcal{L} i e_{1}\left(i_{t}\right)$ is of the form $L_{1} \odot \cdots \odot L_{t}$, with $L_{i} \in \mathcal{L} i e_{1}$, for $i=1, \ldots, t$; and where $\odot$ denotes the commutative tensor product, that is the quotient of the tensor product under the permutation of terms.

Proposition 2.24 Under the isomorphism of operads $\mathcal{G} \cong \mathcal{C}$ om $\circ \mathcal{L} i e_{1}$, the structure of $D$-operad on the right-hand side is given by

$$
\delta \cdot\left(L_{1} \odot \cdots \odot L_{t}\right)=\sum_{1 \leq i<j \leq n}(-1)^{\varepsilon_{i, j}}\left\langle L_{i} ; L_{j}\right\rangle \odot L_{1} \odot \cdots \odot \widehat{L}_{i} \odot \cdots \odot \hat{L}_{j} \odot \cdots \odot L_{t}
$$

where $\left\langle L_{i} ; L_{j}\right\rangle:=\gamma_{\mathcal{L i e}_{1}}\left(\langle-;-\rangle ; L_{i}, L_{j}\right)$ and where the sign $\varepsilon_{i, j}$, arising from the Koszul sign rule, is given by

$$
\varepsilon_{i, j}=\left(\left|L_{i}\right|+\left|L_{j}\right|\right)\left(\left|L_{1}\right|+\cdots+\left|L_{i-1}\right|\right)+\left|L_{j}\right|\left(\left|L_{i+1}\right|+\cdots+\left|L_{j-1}\right|\right) .
$$

Proof For $t=2$, a representative of $L_{1} \odot L_{2}$ is given by $\bullet \otimes L_{1} \otimes L_{2}$, with $L_{1}, L_{2} \in \mathcal{L i e}_{1}$. Since the bracket is sent to 0 under the action of $\delta$, so is any element of $\mathcal{L i e}_{1}$. Thus, we get

$$
\delta \cdot\left(\bullet \otimes L_{1} \otimes L_{2}\right)=\langle-;-\rangle \otimes L_{1} \otimes L_{2}=\left\langle L_{1} ; L_{2}\right\rangle .
$$

We conclude the proof by induction on $t \geq 2$. 
Remark The action of $\delta$ on an element $L_{1} \odot \cdots \odot L_{t}$ in $\mathcal{C} o m \circ \mathcal{L} i e_{1}$ is exactly the image of $\delta^{*} \otimes L_{1} \odot \cdots \odot L_{t}$ under the derivation ${ }^{t} d_{\varphi}$, which is given by Galvez-Carillo, Tonks and Vallette [10, Proof of Lemma 5]. In particular, $\delta$ acts as the ChevalleyEilenberg boundary maps defining the homology of Lie algebras.

Proposition 2.25 The category of $D-\mathcal{G}$-algebras is isomorphic to the category of BV -algebras.

Proof It is a straightforward consequence of Proposition 2.19 conjugated with the following isomorphism of operads proved by Salvatore and Wahl in [24]:

$$
\mathcal{B} \mathcal{V} \cong \mathcal{G} \rtimes D .
$$

Remark The algebraic structure of a Batalin-Vilkovisky algebra can be encoded in two different ways, using the operad $\mathcal{B V}$ or the $D$-operad $\mathcal{G}$. When using the graded $D$-operad $\mathcal{G}$, the unary operator $\Delta$ is provided by the underlying category of mixed chain complexes and its relations with the product and the Lie bracket are encoded in the action of $\delta$ on those generating operations.

More generally, we can consider algebras over the homology of the framed little $n-$ discs operad $f \mathcal{D}_{n}$. Batalin-Vilkovisky algebras correspond to the case $n=2$, by Getzler [11]. In this case, Poisson $n$-algebras, that is algebras over the homology of the little $n$-discs operad $\mathcal{D}_{n}$, play the role of Gerstenhaber algebras. In the same way, it is proved in [24] that

$$
f \mathcal{D}_{n} \cong \mathcal{D}_{n} \rtimes \mathrm{SO}(n),
$$

as topological operads. Hence, taking the homology, we obtain that

$$
\mathrm{H}\left(f \mathcal{D}_{n}\right) \cong \mathrm{H}\left(\mathcal{D}_{n}\right) \rtimes \mathrm{H}(\mathrm{SO}(n)) .
$$

In other words, a structure of algebra over $\mathrm{H}\left(f \mathcal{D}_{n}\right)$ is equivalent to a Poisson $n$-algebra structure in the category of $\mathrm{H}(\mathrm{SO}(n))$-modules.

However, when $n>3$, the algebra $\mathrm{H}(\mathrm{SO}(n))$ has more than one generator. So, we can choose to keep some in the operad and to put only a part of these generators in the underlying category. We still have a Hopf algebra, which acts on the operad thus obtained.

2.3.3 Differential graded algebras over an operad Let $\mathcal{P}$ be an operad. A differential graded $\mathcal{P}$-algebra is a chain complex $\left(A, d_{A}\right)$ endowed with a $\mathcal{P}$-algebra structure such that $d_{A}$ is a derivation for all the operations of $\mathcal{P}$. We can encode this structure in the $D$-operad $\mathcal{P}$, with the trivial $D$-action. In particular, for $\mathcal{P}=\mathcal{A} s s$, the semidirect product $\mathcal{A} s \rtimes D$ is isomorphic to the operad encoding differential graded algebras, 
considered by Livernet, Roitzheim and Whitehouse [20] when the bicomplexes are concentrated in horizontal degree 0 .

\section{Relative Koszul duality theory}

In this section, we develop the Koszul duality theory for operads over a cocommutative Hopf algebra $H$, following the method of Loday and Vallette [21, Chapter 7]. At each step, we prove that the objects can be enriched with an action of $H$, and we show that the results still hold in this context.

Let $\left(\mathcal{P}, \gamma, \eta, d_{\mathcal{P}}\right)$ be a $\mathrm{dg} H$-operad and $\left(\mathcal{C}, \Delta, \eta, d_{\mathcal{C}}\right)$ be a dg $H$-cooperad.

\subsection{Twisting morphisms}

We consider the following dg $\mathbb{S}-H$-module

$$
\left(\operatorname{Hom}(\mathcal{C}, \mathcal{P}):=\left\{\operatorname{Hom}_{\mathbb{K}}(\mathcal{C}(n), \mathcal{P}(n))\right\}_{n \geq 0}, \partial\right),
$$

where $\mathbb{S}_{n}$ acts by conjugation and where $\partial(f)=\left[d_{\mathcal{P}}, f\right]:=d_{\mathcal{P}} \circ f-(-1)^{|f|} f \circ d_{\mathcal{C}}$.

Proposition 3.1 The $d g \mathbb{S}-H$-module $(\operatorname{Hom}(\mathcal{C}, \mathcal{P}), \partial)$ is a $d g H$-operad, called the convolution $H$-operad.

Proof By Berger and Moerdijk [3, Section 1], the $\mathbb{S}-$ module $(\operatorname{Hom}(\mathcal{C}, \mathcal{P})$, $\partial)$ is an operad with respect to the composition map

$$
\gamma_{\text {Hom }(\mathcal{C}, \mathcal{P})}\left(f ; g_{1}, \ldots, g_{k}\right):=\gamma_{\mathcal{P}} \circ\left(f \otimes g_{1} \otimes \cdots \otimes g_{k}\right) \circ \Delta_{\mathcal{C}}
$$

Moreover, in [21, Section 6.4.1], it is proved that $\left(\operatorname{Hom}(\mathcal{C}, \mathcal{P}), \gamma_{\operatorname{Hom}(\mathcal{C}, \mathcal{P})}, \partial\right)$ is a $\mathrm{dg}$ operad. The map $\gamma_{\operatorname{Hom}(\mathcal{C}, \mathcal{P})}$ is $H$-equivariant as a composite of $H$-equivariant maps. And, since $d_{\mathcal{P}}$ and $d_{\mathcal{C}}$ are $H$-equivariant, then so is the differential $\partial$.

Recall that the following composite defines a pre-Lie product on $\prod_{n \geq 0} \operatorname{Hom}(\mathcal{C}, \mathcal{P})(n)$ :

$$
f \star g:=\mathcal{C} \stackrel{\Delta_{(1)}}{\longrightarrow} \mathcal{C} \circ_{(1)} \mathcal{C} \stackrel{f \circ_{(1)}^{g}}{\longrightarrow} \mathcal{P} \circ_{(1)} \mathcal{P} \stackrel{\gamma_{(1)}}{\longrightarrow} \mathcal{P} \text {. }
$$

We denote by $\operatorname{Hom}_{H-\mathbb{S}_{n}}(\mathcal{C}(n), \mathcal{P}(n))$ the space of $H$ - $\mathbb{S}_{n}$-bimodule morphisms from $\mathcal{C}(n)$ to $\mathcal{P}(n)$ and we denote the associated product of $\mathbb{S}-H$-equivariant maps by

$$
\operatorname{Hom}_{\mathbb{S}-H}(\mathcal{C}, \mathcal{P}):=\prod_{n \geq 0} \operatorname{Hom}_{H-\mathbb{S}_{n}}(\mathcal{C}(n), \mathcal{P}(n)) .
$$


Proposition 3.2 The space $\left(\operatorname{Hom}_{\mathbb{S}-H}(\mathcal{C}, \mathcal{P}), \star, \partial\right)$ is a dg pre-Lie algebra. The associated Lie bracket induces a dg Lie algebra structure $\left(\operatorname{Hom}_{\mathbb{S}-H}(\mathcal{C}, \mathcal{P}),[-,-], \partial\right)$.

Proof By [21, Proposition 6.4.5, Lemma 6.4.6], it only remains to prove that if $f$ and $g$ are in $\operatorname{Hom}_{\mathbb{S}-H}(\mathcal{C}, \mathcal{P})$ then so is $f \star g$. This is the case since the pre-Lie product $\star$ is defined to be the composite of $H$-equivariant maps.

Consider the Maurer-Cartan equation in the dg-pre-Lie algebra $\left(\operatorname{Hom}_{\mathbb{S}-H}(\mathcal{C}, \mathcal{P}), \star, \partial\right)$

$$
\partial(\alpha)+\alpha \star \alpha=0 .
$$

Definition 3.3 A solution $\alpha: \mathcal{C} \rightarrow \mathcal{P}$ of degree -1 to the Maurer-Cartan equation is called a twisting $H$-morphism. We denote by $\operatorname{Tw}_{H}(\mathcal{C}, \mathcal{P})$ the space of twisting $H$-morphisms from $\mathcal{C}$ to $\mathcal{P}$.

When $\mathcal{C}$ is a coaugmented $\mathrm{dg}$ cooperad and $\mathcal{P}$ is an augmented $\mathrm{dg}$ operad, we require that the composition of a twisting morphism with respectively the coaugmentation map or the augmentation map vanishes.

\subsection{Bar and cobar constructions}

Are the two functors $\operatorname{Tw}_{H}(\mathcal{C},-)$ and $\operatorname{Tw}_{H}(-, \mathcal{P})$ representable?

Recall from [21, Section 6.5], that the bar construction of an augmented dg operad $\mathcal{P}$ is the dg conilpotent cooperad

$$
\mathrm{B} \mathcal{P}:=\left(\mathcal{T}^{c}(s \overline{\mathcal{P}}), d_{\mathrm{B} \mathcal{P}}\right),
$$

and that the cobar construction of a coaugmented $\mathrm{dg}$ cooperad $\mathcal{C}$ is the dg augmented operad

$$
\Omega \mathcal{C}:=\left(\mathcal{T}\left(s^{-1} \overline{\mathcal{C}}\right), d_{\Omega \mathcal{C}}\right)
$$

Proposition 3.4 Let $\mathcal{P}$ be an $H$-operad and $\mathcal{C}$ be an $H$-cooperad. Then, the $H-$ module structure of the free operad over an $\mathbb{S}-H$-module makes $\mathrm{B} \mathcal{P}$ and $\Omega \mathcal{C}$ into a $d g$ $H$-cooperad and a dg $H$-operad respectively.

Proof By Proposition 2.15, the free operad $\mathcal{T}\left(s^{-1} \overline{\mathcal{C}}\right)$ is an $H$-operad. The differential $d_{\Omega \mathcal{C}}$ is equal to the sum $d_{1}+d_{2}$, where $d_{1}$ is the unique derivation which extends

$$
\varphi: s^{-1} \overline{\mathcal{C}} \stackrel{\text { id } \otimes d_{\mathcal{C}}}{\longrightarrow} \mathcal{T}\left(s^{-1} \overline{\mathcal{C}}\right),
$$


and where $d_{2}$ is the unique derivation which extends

$$
\begin{aligned}
\psi:\left(\mathbb{K} s^{-1} \otimes \overline{\mathcal{C}}\right) & \stackrel{\Delta_{s} \otimes \Delta_{(1)}}{\longrightarrow}\left(\mathbb{K} s^{-1} \otimes \mathbb{K} s^{-1}\right) \otimes\left(\overline{\mathcal{C}}{ }_{(1)} \overline{\mathcal{C}}\right) \\
& \stackrel{\operatorname{Id} \otimes \tau \otimes \operatorname{Id}}{\longrightarrow}\left(\mathbb{K} s^{-1} \otimes \overline{\mathcal{C}}\right)^{\circ}{ }_{(1)}\left(\mathbb{K} s^{-1} \otimes \overline{\mathcal{C}}\right) \cong \mathcal{T}\left(s^{-1} \overline{\mathcal{C}}\right)^{(2)} \succ \mathcal{T}\left(s^{-1} \overline{\mathcal{C}}\right) .
\end{aligned}
$$

The result [21, Proposition 6.3.15] extends to graded $\mathbb{S}-H$-modules. Indeed, if $E$ is an $\mathbb{S}-H$-module and $\alpha: E \rightarrow \mathcal{T}(E)$ is a morphism of $\mathbb{S}-H$-modules, the unique derivation which extends $\alpha$ is a composite of $H$-equivariant maps. Thus, since $\varphi$ and $\psi$ are morphisms of $\mathbb{S}-H$-modules, the derivations $d_{1}$ and $d_{2}$, and hence $d_{\Omega \mathcal{C}}$, are $H$-equivariant maps.

In the same way, we prove that $\mathrm{B} \mathcal{P}$ is a $\mathrm{dg} H$-cooperad.

Proposition 3.5 The bar and cobar constructions form a pair of adjoint functors

$\Omega:\{$ conil. dg $H$-cooperads $\} \rightleftharpoons\{$ aug. dg $H$-operads $\}: \mathrm{B}$,

such that the adjunction is given by the set of twisting $H$-morphisms. That is for every augmented $d g H$-operad $\mathcal{P}$ and every conilpotent $d g H$-cooperad $\mathcal{C}$, there exist natural bijections

$$
\operatorname{Hom}_{\mathrm{dgOp}_{H}}(\Omega \mathcal{C}, \mathcal{P}) \cong \mathrm{Tw}_{H}(\mathcal{C}, \mathcal{P}) \cong \operatorname{Hom}_{\mathrm{dgCoop}_{H}}(\mathcal{C}, \mathrm{B} \mathcal{P})
$$

Proof It is a consequence of [21, Theorem 6.5.10] and of the definitions of the free $H$-operad and the cofree $H$-cooperad over an $\mathbb{S}-H$-module.

We denote by $\eta_{\mathcal{C}}: \mathcal{C} \rightarrow \Omega \mathcal{C}$ and $\pi_{\mathcal{P}}: \mathrm{B} \mathcal{P} \rightarrow \mathcal{P}$ the universal twisting $H$-morphisms corresponding respectively to the unit $v: \mathcal{C} \rightarrow \mathrm{B} \Omega \mathcal{C}$ and to the counit $\epsilon: \Omega \mathrm{B} \mathcal{P} \rightarrow \mathcal{P}$ of the adjunction.

Proposition 3.6 Any twisting $H$-morphism $\alpha: \mathcal{C} \rightarrow \mathcal{P}$ factors uniquely through the universal twisting morphisms $\pi_{\mathcal{P}}$ and $\eta_{\mathcal{C}}$ as follows:

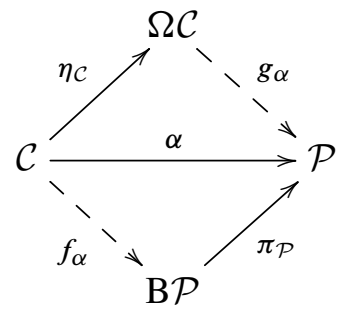

where $g_{\alpha}$ is a morphism of $d g H$-operads and $f_{\alpha}$ is a morphism of $d g H$-cooperads. 
Proof It is a consequence of the adjunction given in Proposition 3.5.

When dealing with operads in the monoidal category of $\mathrm{dg} \mathbb{S}$-modules, a twisting morphism $\alpha$ in $\operatorname{Tw}(\mathcal{C}, \mathcal{P})$ is called $K o s z u l$ when a certain chain complex, called the Koszul complex, is acyclic; see [21, Section 6.6.1].

Definition 3.7 A twisting $H$-morphism $\alpha \in \operatorname{Tw}_{H}(\mathcal{C}, \mathcal{P})$ is said to be $K o s z u l$ when, seen as a morphism of dg $\mathbb{S}$-modules, it is a Koszul morphism.

So, the property for a twisting morphism to be Koszul is a homological property and only depends on the differential structures of $\mathcal{C}$ and $\mathcal{P}$. By definition, to be a quasi-isomorphism is also a homological property, so [21, Theorem 6.6.2] extends to $H$-operads to give the following result.

Theorem 3.8 Let $\mathcal{P}$ be a connected weight graded $d g H$-operad and let $\mathcal{C}$ be a connected weight graded dg $H$-cooperad. Let $\alpha: \mathcal{C} \rightarrow \mathcal{P}$ be a twisting $H$-morphism. The following assertions are equivalent:

(1) The twisting morphism $\alpha$ is Koszul.

(2) The morphism of $d g H$-cooperads $f_{\alpha}: \mathcal{C} \rightarrow \mathrm{B} \mathcal{P}$ is a quasi-isomorphism.

(3) The morphism of $d g H$-operads $g_{\alpha}: \Omega \mathcal{C} \rightarrow \mathcal{P}$ is a quasi-isomorphism.

Theorem 3.9 The counit $v: \Omega \mathrm{B} \mathcal{P} \rightarrow \mathcal{P}$ and the unit $\epsilon: \mathcal{C} \rightarrow \mathrm{B} \Omega \mathcal{C}$ of the adjunction are $H$-quasi-isomorphisms of $d g H$-operads and $d g H$-cooperads respectively.

The resolution $\Omega \mathrm{B} \mathcal{P}$ is called the bar-cobar resolution of $\mathcal{P}$.

\subsection{Koszul duality of $\boldsymbol{H}$-operads}

Definition 3.10 A quadratic $H$-datum consists of a pair $(E, R)$ made up of a graded $\mathbb{S}-H$-module $E$ and a graded sub- $\mathbb{S}-H$-module $R$ of $\mathcal{T}(E)^{(2)}$ called respectively the generating operations and the relations.

The quadratic $H$-operad $\mathcal{P}(E, R)$ associated to a quadratic $H$-datum $(E, R)$ is the quotient $H$-operad of $\mathcal{T}(E)$, which satisfies the following universal property: for any $H$-operad $\mathcal{P}$ of $\mathcal{T}(E)$ such that the composite of morphisms of $\mathbb{S}-H$-modules

$$
R \succ \mathcal{T}(E) \rightarrow \mathcal{P}
$$


is trivial, there exists a unique morphism of $\mathrm{H}$-operads which makes the following diagram commutative:

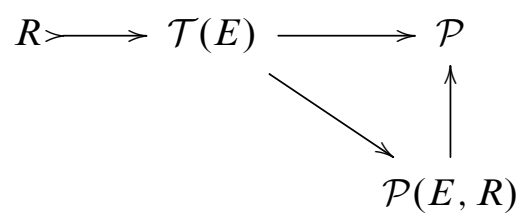

Dually, we define the quadratic $H$-cooperad $\mathcal{C}(E, R)$ associated to a quadratic $H$ datum $(E, R)$.

Proposition 3.11 Let $(E, R)$ be a quadratic $H$-datum. The quadratic $H$-operad (resp. $H$-cooperad) associated to $(E, R)$ is given by the quadratic operad (resp. cooperad) associated to $(E, R)$ in the category of $\mathbb{S}$-modules, endowed with the $H$-module structure induced by the one on the $\mathbb{S}-H$-module $\mathcal{T}(E)$.

Proof We only prove this result for the quadratic $H$-operad. In the category of operads, $\mathcal{P}(E, R)$ is given by the quotient $\mathcal{T}(E) /(R)$ of the free operad on $E$ by the ideal generated by $R$. By Proposition $2.16, \mathcal{T}(E) /(R)$ is an $H$-operad and, by assumptions on $R$ and on $\mathcal{P}$, the morphism of operads $\mathcal{P}(E, R) \rightarrow \mathcal{P}$ turns out to be an $H$-equivariant map.

We can extend the definition of the Koszul dual (co)operad, given in [21, Section 7.2], to the category of $\mathbb{S}-H$-modules. In the classical case, recall that the Koszul dual cooperad of quadratic operad $\mathcal{P}$ is defined by the cooperad

$$
\mathcal{P}^{i}:=\mathcal{C}\left(s E, s^{2} R\right),
$$

and the Koszul dual operad of $\mathcal{P}$ by the operad

$$
\mathcal{P}^{!}:=\left(\mathcal{S}^{c} \underset{\mathcal{H}}{\otimes} \mathcal{P}^{i}\right)^{*},
$$

where ${ }^{*}$ denotes the aritywise linear dual; see Section 2.2.5.

Proposition 3.12 If $\mathcal{P}$ is a quadratic $H$-operad, then the Koszul dual cooperad (resp. operad) of $\mathcal{P}$ is an $H$-cooperad (resp. $H$-operad).

Proof By definition, $\mathcal{P} i$ is an $H$-cooperad. Since $\Delta_{\text {End }} s$ is $H$-equivariant, the $H$-operad structure on $\mathcal{P}^{!}$follows from Proposition 2.21 and Section 2.2.5. 
Proposition 3.13 Let $(E, R)$ be a quadratic $H$-datum such that $E$ is a finite dimensional $\mathbb{S}-H$-module. The Koszul dual operad $\mathcal{P}^{!}$of the quadratic operad $\mathcal{P}=\mathcal{P}(E, R)$ admits the quadratic presentation

$$
\mathcal{P}^{!} \cong \mathcal{P}\left(s^{-1} \mathcal{S}^{-1} \underset{\mathcal{H}}{\otimes} E^{*}, R^{\perp}\right)
$$

where $R^{\perp}$ denotes the sub-S $-H$-module obtained by proper suspension of the operations indexing the vertices of the trees of the orthogonal module $\left(s^{2} R\right)^{\perp} \subset \mathcal{T}\left(s^{-1} E^{*}\right)^{(2)}$, which is the image of $\left(s^{2} R\right)^{*}$ under the isomorphism $\left(\mathcal{T}(E)^{(2)}\right)^{*} \cong \mathcal{T}\left(s^{-1} E^{*}\right)^{(2)}$.

Proof By [21, Proposition 7.2.4], this result is true in the category of operads. It is left to prove that the isomorphism is an $H$-equivariant map: the restriction of this isomorphism to the space of generators $s^{-1} \mathcal{S}^{-1} \underset{\mathcal{H}}{\otimes E^{*}}$ is $H$-equivariant, thus so is the isomorphism.

Proposition 3.14 Let $(E, R)$ be a quadratic $H$-datum and $\mathcal{P}=\mathcal{P}(E, R)$ be its associated quadratic $H$-operad. The natural $H$-cooperad inclusion $i: \mathcal{P} i \hookrightarrow \mathrm{B} \mathcal{P}$ induces an isomorphism of graded $H$-operads

$$
i: \mathcal{P}^{i} \stackrel{\cong}{\rightarrow} \mathrm{H}^{0}\left(\mathrm{~B}^{*} \mathcal{P}\right),
$$

where $\mathrm{H}^{\bullet}\left(\mathrm{B}^{*} \mathcal{P}\right)$ denotes the cohomology groups of the syzygy degree cochain complex associated to $\mathrm{B} \mathcal{P}$; see [21, Section 7.3.1].

Proof By [21, Proposition 7.3.2], we have an isomorphism of operads and the inclusion $\mathcal{P} \mathrm{i} \hookrightarrow \mathrm{B}^{0} \mathcal{P}$ is exactly the kernel of the differential defining $\mathrm{H}^{\bullet}\left(\mathrm{B}^{*} \mathcal{P}\right)$, that is $\mathrm{H}^{0}\left(\mathrm{~B}^{*} \mathcal{P}\right)$. Since this differential is $H$-equivariant, this isomorphism is compatible with the $H$ module structure.

Lemma 3.15 Let $(E, R)$ be a quadratic $H$-datum. Then, the composite

$$
\kappa: \mathcal{C}\left(s E, s^{2} R\right) \rightarrow s E \stackrel{s^{-1}}{\longrightarrow} E \hookrightarrow \mathcal{P}(E, R)
$$

associated to $(E, R)$, is a twisting $H$-morphism.

Proof It is straightforward from [21, Lemma 7.4.2], since $\kappa$ is a composite of $H$ equivariant maps. 
Theorem 3.16 (Koszul criterion) Let $(E, R)$ be a quadratic $H$-datum and $\mathcal{P}=$ $\mathcal{P}(E, R)$ be its associated quadratic $H$-operad. The following statements are equivalent:

(1) The inclusion $i: \mathcal{P} i \succ \mathrm{B} \mathcal{P}$ is a quasi-isomorphism of dg $H$-cooperads.

(2) The projection $p: \Omega \mathcal{P}^{i} \rightarrow \mathcal{P}$ is a quasi-isomorphism of $d g H$-operads.

When these propositions hold, we say that $\mathcal{P}$ is a Koszul operad.

Proof This follows from Theorem 3.8.

\subsection{Example}

Let us now focus on the example of Batalin-Vilkovisky algebras.

Getzler and Jones [13] proved that the operad $\mathcal{G}$ is Koszul. Then, by the present definition, so is the $D$-operad $\mathcal{G}$.

Lemma 3.17 The action of $D$ on $\mathcal{G}^{i}$ is induced by

$$
\begin{aligned}
\delta \cdot: \mathcal{G}^{i} & \rightarrow \mathbb{K} s \bullet \oplus \mathbb{K} s\langle-;-\rangle, \\
s \bullet & \mapsto-s\langle-;-\rangle, \\
s\langle-;-\rangle & \mapsto 0 .
\end{aligned}
$$

Proof By definition, the action of $D$ is characterized by its value on the cogenerators of $\mathcal{G}^{i}$, which is deduced from the one on $\mathcal{G}$.

Proposition 3.18 Under the isomorphism of cooperads $\mathcal{G}^{i} \cong \mathcal{S}^{-1^{c}} \mathcal{C o m}_{1}^{c} \circ \mathcal{S}^{-1}{ }^{c} \mathcal{L i e}^{c}$, the structure of $D$-cooperad on the right-hand side is given by

$$
\delta \cdot\left(L_{1} \odot \cdots \odot L_{t}\right)=\sum_{k=1}^{n}(-1)^{\varepsilon_{k}} L_{1} \odot \cdots \odot L_{k}^{\prime} \odot L_{k}^{\prime \prime} \odot \cdots \odot L_{t},
$$

where $L_{k}^{\prime} \odot L_{k}^{\prime \prime}$ is the sumless $S$ weedler's notation for the image of $L_{k}$ under the binary

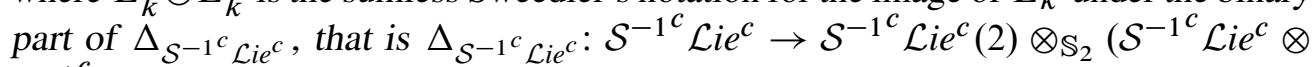
$\left.\mathcal{S}^{-1^{c}} \mathcal{L} i e^{c}\right)$ and

$$
\varepsilon_{k}=\left|L_{k}\right|+\cdots+\left|L_{t}\right|+1 \text {. }
$$


Proof Recall that, for any $\mathbb{S}-H$-module $M, \mathcal{S}^{-1^{c}} M$ (resp. $\mathcal{S} M$ ) denotes the Hadamard product of $\mathcal{S}^{-1^{c}}$ (resp. $\mathcal{S}$ ) with $M$. To make explicit the action of $\delta$, we use the $D$-operad structure on $\left(\mathcal{G}^{i}\right)^{*} \cong \mathcal{S C o m}{ }_{-1} \circ \mathcal{S} \mathcal{L}$ ie, which is given by the isomorphism of operads $\left(\mathcal{G}^{i}\right)^{*} \cong \mathcal{S}^{2} \mathcal{G} \cong \mathcal{S}^{2}\left(\mathcal{C o m} \circ \mathcal{L}_{i} e_{1}\right)$. We have

$$
\delta \cdot\left(L_{1} \odot \cdots \odot L_{t}\right)=\delta \cdot\left(L_{1}^{*} \odot \cdots \odot L_{t}^{*}\right)^{*},
$$

with $L_{1}^{*} \odot \cdots \odot L_{t}^{*} \in \mathcal{S C}$ om $_{-1} \circ \mathcal{S L i e}$. The only elements in $\mathcal{S C o m}$ o $_{1} \circ \mathcal{S} \mathcal{L}$ ie whose images under $\delta \cdot\left(L_{1}^{*} \odot \cdots \odot L_{t}^{*}\right)^{*}$ are nonzero are of the form

$$
L_{1}^{*} \odot \cdots \odot\left(L_{k}^{\prime}\right)^{*} \odot\left(L_{k}^{\prime \prime}\right)^{*} \odot \cdots \odot L_{t}^{*}, \quad k \in\{1, \ldots, t\},
$$

where $\left(L_{k}^{\prime}\right)^{*},\left(L_{k}^{\prime \prime}\right)^{*}$ are elements of $\mathcal{S} \mathcal{L}$ ie such that $L_{k}^{*}=\gamma_{\mathcal{S} \mathcal{L} i e}\left(s^{-1}[-;-] ;\left(L_{k}^{\prime}\right)^{*},\left(L_{k}^{\prime \prime}\right)^{*}\right)$. This image is equal to $(-1)^{\varepsilon_{k}+1}$, where $\varepsilon_{k}=\left|L_{k}^{*}\right|+\cdots+\left|L_{t}^{*}\right|$.

Remark Dually to the $D$-operad structure on $\mathcal{G}$, the $D$-cooperad structure on $\mathcal{G}^{i}$ is equal, up to sign, to the image of an element in $\mathbb{K}[\delta]_{1} \circ \mathcal{S}^{-1}{ }^{c} \mathcal{C o m}_{1}^{c} \circ \mathcal{S}^{-1^{c}} \mathcal{L i e}^{c}$ under the coderivation $d_{\varphi}$, given by Galvez-Carillo, Tonks and Vallette [10, Lemma 5].

\section{Homotopy algebras and transfer theorem}

In this section, we extend some definitions and results of [21, Section 10] to the category of $H$-modules, requiring in addition the compatibility with the $H$-module structure.

\subsection{The category of homotopy algebras}

Let $\mathcal{P}$ be a Koszul $H$-operad. By definition, a homotopy $H$-P-algebra, or an $H-\mathcal{P}_{\infty}-$ algebra, is an algebra over the Koszul resolution $\mathcal{P}_{\infty}:=\Omega \mathcal{P} i$ of $\mathcal{P}$ in the category of $H$-operads. So, a structure of algebra over $\mathcal{P}_{\infty}$ on an $H$-module $A$ is equivalent to a morphism of $H$-operads $\mathcal{P}_{\infty}:=\Omega \mathcal{P}^{i} \rightarrow \operatorname{End}_{A}$. Notice that a $H-\mathcal{P}_{\infty}$-algebra is a $\mathcal{P}_{\infty}$-algebra endowed with a compatible $H$-module structure.

Example Any $H$-P -algebra structure on an $H$-module is a particular case of a $H-\mathcal{P}_{\infty}$-algebra structure.

Proposition 4.1 The set of $H-\mathcal{P}_{\infty}$-algebra structures is equivalently given by

$$
\begin{aligned}
\operatorname{Hom}_{\mathrm{dg} H-\mathrm{Op}}\left(\Omega \mathcal{P}^{i}, \operatorname{End}_{A}\right) & \cong \operatorname{Tw}_{H}\left(\mathcal{P}^{i}, \operatorname{End}_{A}\right) \\
& \cong \operatorname{Hom}_{\mathrm{dg}} H-\operatorname{coOp}\left(\mathcal{P}^{i}, \operatorname{B~End}_{A}\right) \cong \operatorname{Codiff}_{H}\left(\mathcal{P}^{i}(A)\right),
\end{aligned}
$$

where $\operatorname{Codiff}_{H}\left(\mathcal{P}^{i}(A)\right)$ denotes the set of codifferentials on $\mathcal{P}^{i}(A)$, that are $H-$ equivariant coderivations on $\mathcal{P}^{i}(A)$ squaring to zero. 
Proof The first two bijections are given by the adjunction of Proposition 3.5. The proof of Proposition 2.18 implies that

$$
\operatorname{Hom}_{\mathbb{S}-H-\operatorname{Mod}}\left(\mathcal{P}^{\mathrm{i}}, \operatorname{End}_{A}\right) \cong \operatorname{Hom}_{H}\left(\mathcal{P}^{\mathrm{i}}(A), A\right) .
$$

Moreover, [21, Proposition 6.3.17] extends to $H$-modules. So, if $\mathcal{C}$ is a dg $H$-cooperad, $V$ is an $H$-module and $\alpha: \mathcal{C}(V) \rightarrow V$ is $H$-equivariant, then the unique coderivation of the cofee $\mathcal{C}$-coalgebra $\mathcal{C}(V)$ which extends $\alpha$ is given by a sum of composites of $H$-equivariant maps. Thus, we get the isomorphism

$$
\operatorname{Hom}_{H}\left(\mathcal{P}^{\mathrm{i}}(A), A\right) \cong \operatorname{Coder}_{H}\left(\mathcal{P}^{\mathrm{i}}(A)\right) \text {. }
$$

We conclude with [21, Proposition 10.1.19], that an element in $\operatorname{Hom}_{\mathbb{S}-H-\operatorname{Mod}}\left(\mathcal{P}^{i}\right.$, End $\left.V\right)$ is a solution to the Maurer-Cartan equation if and only if the associated coderivation on $\mathcal{P i}(V)$ squares to zero, which does not depend on the $H$-module structure.

This result provides us with four equivalent definitions of $H-\mathcal{P}_{\infty}$-algebra structures. Thus, when dealing with this algebraic structure, we can make an ad hoc choice of one of those definitions.

By extension of the classical definition of [21, Section 10.2.2], an $\infty-H$-morphism of $H-\mathcal{P}_{\infty}$-algebras is a morphism $A \rightsquigarrow B$ of dg $H-\mathcal{P} i$-coalgebras

$$
F:\left(\mathcal{P}^{\mathrm{i}}(A), d_{\varphi}\right) \rightarrow\left(\mathcal{P}^{\mathrm{i}}(B), d_{\psi}\right),
$$

where $\varphi$ and $\psi$ are the twisting $H$-morphisms defining the structure of $H-\mathcal{P}_{\infty}$-algebra on $A$ and $B$ respectively. We denote by $\infty-\mathcal{P}_{\infty}-H$-alg the category of $H-\mathcal{P}_{\infty^{-}}$ algebras and their $\infty-H$-morphisms, where the composite of two $\infty-H$-morphisms is defined as the composite of the associated morphisms of $\mathrm{dg} H-\mathcal{P} i$-coalgebras.

Equivalently, an $\infty-H$-morphism of $H-\mathcal{P}_{\infty}$-algebras is an $H$-equivariant $\infty$-morphism of the underlying $\mathcal{P}_{\infty}$-algebras. A $\infty-H$-morphism of $H-\mathcal{P}_{\infty}$-algebras is called an $\infty-H$-isomorphism (resp. $\infty-H$-quasi-isomorphism) if its first component $A \rightarrow B$ is an $H$-isomorphism (resp. quasi-isomorphism).

\subsection{Homotopy transfer theorem}

Let $\left(B, d_{B}\right)$ be a homotopy retract of $\left(A, d_{A}\right)$ in the category of $H$-modules, that is a homotopy retract of chain complexes

$$
\begin{gathered}
h C\left(A, d_{A}\right) \stackrel{p}{\underset{i}{\rightleftarrows}}\left(B, d_{B}\right), \\
\operatorname{Id}_{A}-i p=d_{A} h+h d_{A},
\end{gathered}
$$


such that the maps $i, p, h$ are $H$-equivariant and $i$ is a quasi-isomorphism.

Theorem 4.2 Let $\mathcal{P}$ be a Koszul H-operad. Let $\left(B, d_{B}\right)$ be a homotopy retract of $\left(A, d_{A}\right)$ in the category of $d g-H$-modules.

Any $H-\mathcal{P}_{\infty}$-algebra structure on $A$, defined by generating operations $\left\{m_{\mu}: A^{\otimes n} \rightarrow\right.$ $\left.A, \mu \in \mathcal{P}_{\infty}\right\}$, can be transferred into a $H-\mathcal{P}_{\infty}$-algebra structure on $B$, which extends the transferred operations $p m_{\mu} i^{\otimes n}: B^{\otimes n} \rightarrow B$, and such that $i$ extends to an $\infty-H-$ quasi-isomorphism.

Proof We check each step of the proof of [21, Theorem 10.3.2] extends to $H$-modules. Since the maps $h, i$ and $p$ are $H$-equivariant, then the map $\Psi: \mathrm{B} \operatorname{End}_{A} \rightarrow \mathrm{B} \operatorname{End}_{B}$, defined in [21, Section 10.3.3], is a morphism of $H$-cooperads. To prove that $\Psi$ is compatible with the differential structure, we do not care about the $H$-module structure so it follows from Van der Laan [18, Theorem 5.2]. Then, as a consequence of the barcobar adjunction, the composite $\mathcal{P} i \rightarrow$ B End $_{A} \rightarrow \mathrm{B} \mathrm{End}_{B}$ defines an $H-\mathcal{P}_{\infty}$-algebra structure on $B$. Similarly, to be an $\infty-H$-quasi-isomorphism does not depend on the $H$-module structure therefore, by [21, Theorem 10.3.11], we just have to prove that the map $i_{\infty}$, defined there, commutes with the $H$-module structure. This is the case since it is a composite of such maps.

In particular, the transferred structure and the $\infty-H$-quasi-isomorphism are both given by the explicit tree-wise formulae of [21, Section 10.3.10, Theorem 10.3.6].

Theorem 4.3 Let $\mathcal{P}$ be a Koszul $H$-operad. Let $\left(A, d_{A}\right)$ be a $\mathcal{P} \rtimes H$-algebra and $\left(B, d_{B}\right)$ be a homotopy retract of $\left(A, d_{A}\right)$ in the category of $d g-H$-modules. Then, $B$ inherits a structure of $H-\mathcal{P}_{\infty}$-algebra, which extends the transferred operations, and such that $i$ extends to an $\infty$-quasi-isomorphism of $H-\mathcal{P}_{\infty}$-algebras.

Proof By Proposition 2.19, the $\mathcal{P} \rtimes H$-algebra structure on $A$ is equivalent to an $H-\mathcal{P}$-algebra structure. Then, we apply Theorem 4.2.

Remark When the operad $\mathcal{P} \rtimes H$ is Koszul, one can apply the HTT for operads, but the $(\mathcal{P} \rtimes H)_{\infty}$-algebra structure can be very complex. If we can do the transfer in the category of $H$-modules, then the transferred structure reduces to a much simpler structure. On the other hand, when the operad $\mathcal{P} \rtimes H$ is not Koszul, the classical method to transfer an algebraic structure must be improved. While, if the $H$-operad $\mathcal{P}$ is itself Koszul, then we can apply our version of the HTT, assuming that the homotopy retract is compatible with the $H$-action. 


\subsection{Homotopy theory for algebras over an $\mathrm{H}$-operad}

The results of [21, Chapter 11] extend mutatis mutandis to the framework of $H$-operads. The objects and the maps defined there are compatible with the $H$-module structure. For the homological considerations, the results still hold because they do not depend on the $H$-action. In particular, we obtain the following results.

Theorem 4.4 (Rectification) Let $\mathcal{P}$ be a Koszul $H$-operad. Any $H-\mathcal{P}_{\infty}$-algebra is naturally $\infty-H$-quasi-isomorphic to a $d g H$-P -algebra.

Theorem 4.5 (Equivalence between homotopy categories) Let $\mathcal{P}$ be a Koszul $\mathrm{H}_{-}$ operad. The homotopy category of $d g(H-\mathcal{P}$-algebras is equivalent to the homotopy category of $H-\mathcal{P}$-algebras with their $\infty-H$-morphisms.

Remark In particular, we obtain a new description for the homotopy category of $\mathcal{P} \rtimes H$-algebras, using a simpler class of $\infty$-morphisms than [21, Theorem 11.4.12].

An advantage of this result lies in the "invertibility" of the $\infty-H$-quasi-isomorphisms. Indeed, any $\infty-H$-quasi-isomorphism admits an $\infty-H$-quasi-isomorphism in the opposite direction, as in [21, Theorem 10.4.7], while it is not the case for $H$-quasiisomorphisms. This enables us to prove the following result.

Theorem 4.6 ( $H$-quasi-isomorphism vs $\infty-H$-quasi-isomorphism) Let $\mathcal{P}$ be a Koszul $H$-operad and $A, B$ be two dg $H$-P-algebras. The following assertions are equivalent:

(1) There exists a zigzag of $H$-quasi-isomorphisms of $d g H$-P -algebras:

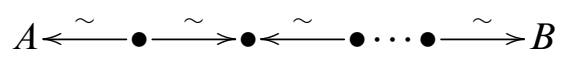

(2) There exists an $\infty-H$-quasi-isomorphism of $d g H-\mathcal{P}$-algebras $A \stackrel{\sim}{\rightsquigarrow} B$.

Remark Since an $H-\mathcal{P}$-algebra structure is equivalent to a $\mathcal{P} \rtimes H$-algebra one, the theorem gives a new and simpler way to prove formality results for $\mathcal{P} \rtimes H$-algebras.

\subsection{Example}

When we encode $\mathrm{BV}$-algebra structures with the operad $\mathcal{B V}$, a notion of homotopy $\mathrm{BV}$-algebra structure is given by Galvez-Carillo, Tonks and Vallette [10]. We describe the homotopy structure obtained when we use the $D$-operad $\mathcal{G}$ instead, and compare these two. 
Proposition 4.7 A homotopy $D-\mathcal{G}$-algebra is a mixed chain complex $\left(A, d_{A}, \Delta_{A}\right)$ endowed with a homotopy Gerstenhaber algebra structure such that

$$
\Delta_{A} m_{p_{1}, \ldots, p_{t}}-(-1)^{t-2} \sum_{i=1}^{n} m_{p_{1}, \ldots, p_{t}} \circ_{i} \Delta_{A}=\sum_{k=1}^{t} \sum_{p_{k}^{\prime}+p_{k}^{\prime \prime}=p_{k}}(-1)^{\varepsilon_{k}+1} m_{p_{1}, \ldots, p_{k}^{\prime}, p_{k}^{\prime \prime}, \ldots, p_{t}},
$$

where $n=p_{1}+\cdots+p_{t}$ and where $\Delta_{A}: A \rightarrow A$ is the unary operator provided by the $D$-module structure on $A$.

Proof By Proposition 4.1, a structure of $D-\mathcal{G}_{\infty}$-algebra on a mixed chain complex $A$ is given by a twisting $D$-morphism $\mathcal{G}^{i} \rightarrow \operatorname{End}_{A}$. It is a twisting morphism $\mathcal{G}^{i} \rightarrow \operatorname{End}_{A}$ between the underlying $\mathbb{S}$-modules, that is a homotopy Gerstenhaber structure as defined in [10, Proposition 16], which commutes with the action of $D$. This condition is then a consequence of Proposition 3.18, according to the study of homotopy Gerstenhaber algebras done in [10, Section 2.1].

As conjectured at the end of [10, Section 2.4], we have the following relation with the homotopy BV-algebras.

Proposition 4.8 A homotopy BV-algebra structure (as defined in [10, Theorem 20]), such that all the operations are trivial except the maps $m_{p_{1}, \ldots, p_{t}}^{0}$ and the map $m_{1}^{1}$, is equivalent to a $D-\mathcal{G}_{\infty}$-algebra.

Proof Looking at the explicit description of the homotopy BV-algebra structure given in [10, Theorem 20], all the relations become trivial except the relations $R_{p_{1}, \ldots, p_{t}}^{0}$, defining the homotopy Gerstenhaber structure, the relations $R_{p_{1}, \ldots, p_{t}}^{1}$, corresponding to those given in Proposition 4.7, and the relation $R_{1}^{2}$, which means that $m_{1}^{1}$ squares to zero.

From now on, we call a $D-\mathcal{G}_{\infty}$-algebra a strict homotopy $B V$-algebra, since a $D-$ $\mathcal{G}_{\infty}$-algebra is a homotopy $\mathrm{BV}$-algebra such that the operator $\Delta$ strictly satisfies the relations of a $\mathrm{BV}$ operator.

Remark A strict homotopy BV-algebra is a homotopy BV-algebra such that any elements in $\mathcal{B V}^{i}$ (as defined in [10, Section 1]) containing a vertex decorated by $\Delta$, except $\Delta$ itself, acts as zero. If we ask, moreover, that $\Delta$ acts as zero, we get the notion of strongly trivialized homotopy BV-algebras, defined by Drummond-Cole [8]. In that article, the author proved that any strongly trivialized homotopy BV structure on a chain complex induces a structure of homotopy hypercommutative algebra; see Drummond-Cole and Vallette [9], on that complex. Thus, we get another description of the homotopy hypercommutative structure. 
Theorem 4.9 Let $\left(B, d_{B}, \Delta_{B}\right)$ be a homotopy retract of $\left(A, d_{A}, \Delta_{A}\right)$ in the category of mixed chain complexes. If $A$ is endowed with a $B V$-algebra structure (or with a strict homotopy $B V$-algebra structure), then $B$ inherits a structure of strict homotopy $B V$-algebra which extends the naive transferred operations, and such that $i$ extends to an $\infty-D$-quasi-isomorphism of strict homotopy $B V$-algebras.

Proof By Proposition 2.25, $A$ is a $D-\mathcal{G}$-algebra. Then we apply Theorem 4.2.

When the homotopy retract lives in the category of mixed chain complexes, this theorem shows that the transferred homotopy BV-algebra structure reduces to a strict homotopy $\mathrm{BV}$-algebra, ie a homotopy BV-algebra structure without higher homotopies arising from $\Delta$ and its relations with the product and with the bracket.

Proposition 4.10 Let $\left(B, d_{B}\right)$ be a homotopy retract of $\left(A, d_{A}\right)$, in the category of chain complexes, which satisfies the following side conditions:

$$
h i=p h=0 .
$$

If $\left(A, d_{A}, \bullet,\langle-;-\rangle, \Delta\right)$ is a $B V$-algebra structure on $A$ such that

$$
\Delta h+h \Delta=0,
$$

then $B$ inherits a strict homotopy $B V$-algebra structure, induced by the $B V$-algebra structure on $A$, and such that $i$ extends to an $\infty-D$-quasi-isomorphism of strict homotopy $B V$-algebras.

Proof We endow the chain complex $\left(B, d_{B}\right)$ with the unary operator $\widetilde{\Delta}$ induced by $\Delta$, and defined by $\tilde{\Delta}:=p \Delta i$. The condition $\Delta h+h \Delta=0$ implies that $\tilde{\Delta}^{2}=0$ so $\left(B, d_{B}, \tilde{\Delta}\right)$ is a mixed chain complex. Moreover, the side conditions imply that the maps $p$ and $i$ are compatible with $\tilde{\Delta}$. Thus, we have a homotopy retract between $\left(A, d_{A}, \Delta\right)$ and $\left(B, d_{B}, \widetilde{\Delta}\right)$ in the category of mixed chain complexes. We conclude by applying Theorem 4.9.

This proposition is the result which answers the main question raised at the beginning of this paper.

When $B$ consists of the homology groups $\mathrm{H}^{\bullet}(A, d)$ of the complex $(A, d)$, it is always possible to build a homotopy retract which satisfies the side conditions: it is called the Hodge decomposition; see Loday and Vallette [21, Lemma 9.4.7]. More precisely, the chain complex $(A, d)$ splits into a direct sum of graded spaces as

$$
A=H \oplus B \oplus C,
$$


where $B=\operatorname{Ker}(d) \cap \operatorname{Im}(d), H \oplus B=\operatorname{Ker}(d)$ and $H \cong \mathrm{H}^{\bullet}(A, d)$. Then, the homotopy retract is defined as follows:

- $\quad i$ is the inclusion of $H$ in $A$.

- $\quad p$ is the projection of $A$ onto $H$.

- $h$ is equal to 0 on $H \oplus C$ and is equal to $d^{-1}$ on $B$.

In this case, the homotopy retract satisfies the aforementioned side conditions.

Example We consider the following nonunital $\mathrm{dg}$ commutative algebra $A$ generated by

$$
x_{3}, y_{3}, t_{3}, \xi_{4}, \omega_{5}, z_{7}, u_{7} \text { and } v_{8} \text {, }
$$

where the subscript gives the homological degree, such that the product by $u$, by $v$, by $\xi$ and by $\omega$ is equal to zero. The algebra $A$ is finite dimensional and spanned by the elements $x, y, t, \xi, \omega, x y, x t, y t, u, z, v, x z, y z, t z, x y z, x y t$ and $x y z t$. We summarize in the following picture the definition of the differential and of the BV operator on the generators:

\begin{tabular}{|c|c|c|c|c|c|c|}
\hline 3 & 4 & 7 & 9 & 10 & 13 & 16 \\
\hline$x$ & $\xi \stackrel{\Delta}{\mapsto} \omega$ & $x y \stackrel{\Delta}{\longmapsto} u \stackrel{d}{\gtrless} v$ & $x y t$ & $x z$ & $x y z$ & $x y z t$ \\
\hline$y$ & & $x t$ & & $y z$ & & \\
\hline$t$ & & $y t$ & & $z t$ & & \\
\hline
\end{tabular}

where an element is sent to 0 if nothing else is specified.

Proposition 4.11 The aforementioned algebra $(A, d, \Delta)$ is a dg $B V$-algebra. Moreover, its homology $\mathrm{H}^{\bullet}(A, d)$ is endowed with a strict homotopy $B V$-algebra structure, such that the inclusion $\mathrm{H}^{\bullet}(A, d) \hookrightarrow A$ extends to an $\infty$-quasi-isomorphism of strict homotopy $B$ V-algebras.

Proof It is straightforward to prove that $A$ together with $d$ and $\Delta$ forms a dg BValgebra. The Hodge decomposition of $A$ is given by

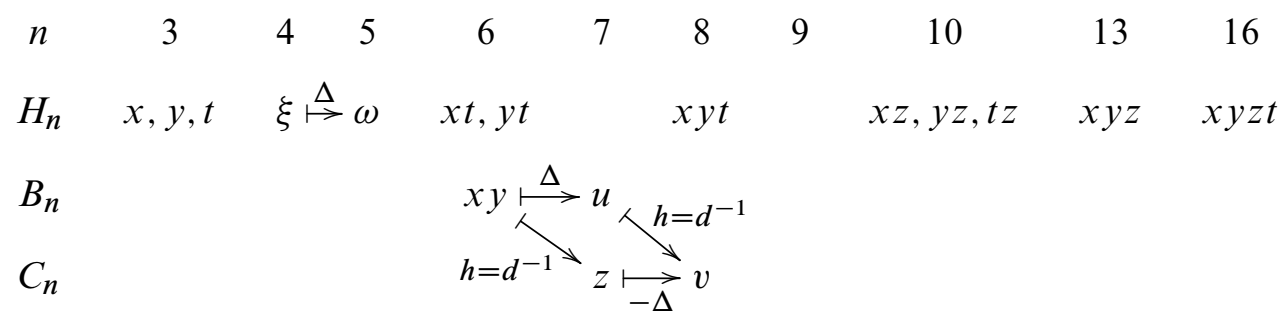


where an element is sent to 0 if nothing else is specified. The contracting homotopy and $\Delta$ commute. Then, we apply Proposition 4.10.

Note that the product induced in homology is associative, since the differential on $\mathrm{H}^{\bullet}(A, d)$ is 0 , but the first homotopy for the associativity relation, given by

$$
m_{3}^{0}:=p((h(i(-) \bullet i(-))) \bullet i(-))-p(i(-) \bullet(h(i(-) \bullet i(-))))
$$

is not equal to 0 . Moreover, the BV operator does not vanish in homology.

Remark Introduced by Deligne, Griffiths, Morgan and Sullivan [7] to study the differential forms of compact Kähler manifolds and used by Barranikov and Kontsevich [1] for the Dolbeault complex of Calabi-Yau manifolds, the $d d^{c}$-lemma implies the condition $[\Delta, h]=0$, but is a strong condition in our setting. Indeed, if the $d d^{c}$-lemma is satisfied, then the operator $\Delta$ vanishes on the homology groups.

Encoding the BV-algebra structure in the $D$-operad $\mathcal{G}$ allows us to deal with a notion of homotopy BV-algebra which is simpler than the one given by Galvez-Carillo, Tonks and Vallette [10]. The associated notion of $\infty$-morphism is also simplified.

Proposition 4.12 An $\infty-D$-morphism of (strict homotopy) $B V$-algebras is an $\infty-$ morphism of the underlying (homotopy) Gerstenhaber algebras which commutes with the extra action of the unary operator, provided by the (strict homotopy) $B V$-algebra structures.

Proof It is a consequence of the definition of an $\infty$-morphism in the category of $D-\mathcal{G}_{\infty}$-algebras.

Corollary 4.13 The homotopy category of $B V$-algebras is equivalent to the homotopy category of strict homotopy $B V$-algebras with their $\infty$-morphisms. Moreover, for any $B V$-algebras $A$ and $B$, the following assertions are equivalent:

(1) There exists a zigzag of quasi-isomorphisms of $B V$-algebras:

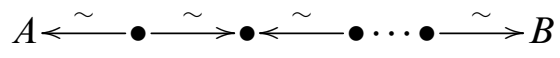

(2) There exists an $\infty$-quasi-isomorphism of Gerstenhaber algebras $A \stackrel{\sim}{\rightsquigarrow} B$, commuting with the unary operator provided by the $B V$-algebra structures.

Proof Since the operad $\mathcal{G}$ is Koszul, we can apply Theorem 4.5 and Theorem 4.6, combined with Proposition 4.12. 
This theorem provides a new way to prove the existence of a chain of quasi-isomorphisms between $\mathrm{BV}$-algebras. In particular, it could help to prove the formality of a BV-algebra. Moreover, we could hope to use it to study the interpretation of the mirror symmetry conjecture of Kontsevich [17] in terms of BV-algebras.

The mirror symmetry conjecture says that a certain A-type theory of a Calabi-Yau manifold can be identified with a B-type theory on a mirror manifold. Cao and Zhou [4] interpret this conjecture as the existence of a quasi-isomorphism of $\mathrm{dg} \mathrm{BV}$-algebras from the De Rham complex of a Calabi-Yau manifold $\mathcal{M}$ to the Dolbeault complex of a dual Calabi-Yau manifold $\widetilde{\mathcal{M}}$ :

$$
\left(\Omega^{n-\bullet}(\mathcal{M}), d_{D R}, \wedge, \Delta,\langle-;-\rangle\right) \stackrel{\sim}{\rightarrow}\left(\Gamma\left(\widetilde{\mathcal{M}}, \wedge \bar{T}_{\widetilde{\mathcal{M}}}^{*} \otimes \wedge^{\bullet} T_{\widetilde{\mathcal{M}}}\right), \bar{\partial}, \wedge, \operatorname{div},\langle-;-\rangle_{S}\right)
$$

Using Corollary 4.13, it is equivalent to the existence of an $\infty$-quasi-isomorphism of the underlying Gerstenhaber algebras, which commutes strictly with the action of the unary operators $\Delta$ and div.

Acknowledgments This paper constitutes a part of the author's PhD thesis. I would like to thank my advisor, Bruno Vallette, for being so receptive and involved. I am grateful for our instructive discussions and for his fruitful ideas. I also would like to thank Joan Millès for his reactivity to my many questions and Jim Stasheff for his comments. The author is supported by the ANR grant HOGT, referenced as ANR-11BS01-0002.

\section{References}

[1] S Barannikov, M Kontsevich, Frobenius manifolds and formality of Lie algebras of polyvector fields, Internat. Math. Res. Notices (1998) 201-215 MR1609624

[2] I A Batalin, G A Vilkovisky, Gauge algebra and quantization, from: "Quantum gravity”, (M A Markov, P C West, editors), Plenum, New York (1984) 463-480 MR746994

[3] C Berger, I Moerdijk, Axiomatic homotopy theory for operads, Comment. Math. Helv. 78 (2003) 805-831 MR2016697

[4] H-D Cao, J Zhou, DGBV algebras and mirror symmetry, from: "First International Congress of Chinese Mathematicians", (L Yang, S-T Yau, editors), AMS/IP Stud. Adv. Math. 20, Amer. Math. Soc. (2001) 279-289 MR1830184

[5] H-D Cao, J Zhou, On quasi-isomorphic DGBV algebras, Math. Ann. 326 (2003) 459-478 MR1992272

[6] M Chas, D Sullivan, String topology arXiv: 9911159

[7] P Deligne, P Griffiths, J Morgan, D Sullivan, Real homotopy theory of Kähler manifolds, Invent. Math. 29 (1975) 245-274 MR0382702 
[8] G C Drummond-Cole, Formal formality of the hypercommutative algebras of low dimensional Calabi-Yau varieties arXiv:1201.6111

[9] G C Drummond-Cole, B Vallette, The minimal model for the Batalin-Vilkovisky operad, Selecta Math. 19 (2013) 1-47 MR3029946

[10] I Gálvez-Carrillo, A Tonks, B Vallette, Homotopy Batalin-Vilkovisky algebras, J. Noncommut. Geom. 6 (2012) 539-602 MR2956319

[11] E Getzler, Batalin-Vilkovisky algebras and two-dimensional topological field theories, Comm. Math. Phys. 159 (1994) 265-285 MR1256989

[12] E Getzler, Two-dimensional topological gravity and equivariant cohomology, Comm. Math. Phys. 163 (1994) 473-489 MR1284793

[13] E Getzler, J D S Jones, Operads, homotopy algebra and iterated integrals for double loop spaces arXiv:9403055

[14] V Ginzburg, M Kapranov, Koszul duality for operads, Duke Math. J. 76 (1994) 203-272 MR1301191

[15] E Hoffbeck, A Poincaré-Birkhoff-Witt criterion for Koszul operads, Manuscripta Math. 131 (2010) 87-110 MR2574993

[16] T V Kadeishvili, The algebraic structure in the homology of an $A(\infty)$-algebra, Soobshch. Akad. Nauk Gruzin. SSR 108 (1982) 249-252 MR720689

[17] M Kontsevich, Homological algebra of mirror symmetry, from: "Proceedings of the International Congress of Mathematicians, Vol. 1, 2”, (S D Chatterji, editor), Birkhäuser, Basel (1995) 120-139 MR1403918

[18] P Van der Laan, Coloured Koszul duality and strongly homotopy operads arXiv: 0312147

[19] B H Lian, G J Zuckerman, New perspectives on the BRST-algebraic structure of string theory, Comm. Math. Phys. 154 (1993) 613-646 MR1224094

[20] M Livernet, C Roitzheim, S Whitehouse, Derived $A_{\infty}$-algebras in an operadic context, Algebr. Geom. Topol. 13 (2013) 409-440 MR3031646

[21] J-L Loday, B Vallette, Algebraic operads, Grundl. Math. Wissen. 346, Springer, Heidelberg (2012) MR2954392

[22] Y I Manin, Frobenius manifolds, quantum cohomology, and moduli spaces, AMS Coll. Publ. 47, Amer. Math. Soc. (1999) MR1702284

[23] M Markl, Distributive laws and Koszulness, Ann. Inst. Fourier (Grenoble) 46 (1996) 307-323 MR1393517

[24] P Salvatore, N Wahl, Framed discs operads and Batalin-Vilkovisky algebras, Q. J. Math. 54 (2003) 213-231 MR1989873

[25] J D Stasheff, Homotopy associativity of H-spaces: I, II, Trans. Amer. Math. Soc. 108, 275-292; ibid. 108 (1963) 293-312 MR0158400 
[26] ME Sweedler, Hopf algebras, Mathematics Lecture Note Series, W. A. Benjamin, New York (1969) MR0252485

[27] B Vallette, Homotopy theory of homotopy algebras (2013) Available at http:// math.unice.fr/ brunov/HomotopyTheory.pdf

Institut de Mathématiques de Toulouse, Université Paul Sabatier 118 route de Narbonne, 31062 Toulouse Cedex 9, France olivia.bellier@math.univ-toulouse.fr http://www.math.univ-toulouse.fr/ obellier

Received: 25 February 2013 Revised: 6 June 2013 